\title{
Microlocal theory of sheaves and Tamarkin's non displaceability theorem
}

\author{
Stéphane Guillermou and Pierre Schapira \\ February 15, 2012
}

\begin{abstract}
This paper is an attempt to better understand Tamarkin's approach of classical non-displaceability theorems of symplectic geometry, based on the microlocal theory of sheaves, a theory whose main features we recall here. If the main theorems are due to Tamarkin, our proofs may be rather different and in the course of the paper we introduce some new notions and obtain new results which may be of interest.
\end{abstract}

\section{Introduction}

In [12, D. Tamarkin gives a totally new approach for treating classical problems of non-displaceability in symplectic geometry. His approach is based on the microlocal theory of sheaves, introduced and systematically developed in [3, 4, 5]. (Note however that the use of the microlocal theory of sheaves also appeared in a related context in [7, 9, 8.)

The aim of this paper was initially to better understand Tamarkin's ideas and to give more accessible proofs by making full use of the tools of [5] and of the recent paper [2]. But when working on this subject, we found some new results which may be of interest. In particular, we make here a systematic study of the category of torsion objects.

Let us first briefly recall the main facts of the microlocal theory of sheaves. Consider a real manifold $M$ of class $C^{\infty}$ and a commutative unital ring $\mathbf{k}$ of finite global dimension. Denote by $\mathrm{D}^{\mathrm{b}}\left(\mathbf{k}_{M}\right)$ the bounded derived category of 
sheaves of $\mathbf{k}$-modules on $M$. In loc. cit. the authors attach to an object $F$ of $\mathrm{D}^{\mathrm{b}}\left(\mathbf{k}_{M}\right)$ its singular support, or microsupport, $\mathrm{SS}(F)$, a closed subset of $T^{*} M$, the cotangent bundle to $M$. The microsupport is conic for the action of $\mathbb{R}^{+}$on $T^{*} M$ and is involutive (i.e., co-isotropic). The microsupport allows one to localize the triangulated category $\mathrm{D}^{\mathrm{b}}\left(\mathbf{k}_{M}\right)$, and in particular to define the category $\mathrm{D}^{\mathrm{b}}\left(\mathbf{k}_{M} ; U\right)$ for an open subset $U \subset T^{*} M$. This theory is "conic", that is, it is invariant by the $\mathbb{R}^{+}$-action and is related to the homogeneous symplectic structure rather than the symplectic structure.

In order to get rid of the homogeneity, a classical trick is to add a variable which replaces it. This trick appears for example in the complex case in [10] where a deformation quantization ring (with an $\hbar$-parameter) is constructed on the cotangent bundle $T^{*} X$ to a complex manifold $X$ by using the ring of microdifferential operators of [11] on $T^{*}(X \times \mathbb{C})$. Coming back to the real setting, denote by $t$ a coordinate on $\mathbb{R}$, by $(t ; \tau)$ the associated coordinates on $T^{*} \mathbb{R}$, by $T_{\{\tau>0\}}^{*}(M \times \mathbb{R})$ the open subset $\{\tau>0\}$ of $T^{*}(M \times \mathbb{R})$ and consider the map

$$
\rho: T_{\{\tau>0\}}^{*}(M \times \mathbb{R}) \rightarrow T^{*} M, \quad(x, t ; \xi, \tau) \mapsto(x ; \xi / \tau) .
$$

Tamarkin's idea is to work in the localized category $\mathrm{D}^{\mathrm{b}}\left(\mathbf{k}_{M \times \mathbb{R}} ;\{\tau>0\}\right)$, the localization of $\mathrm{D}^{\mathrm{b}}\left(\mathbf{k}_{M \times \mathbb{R}}\right)$ by the triangulated subcategory $\mathrm{D}_{\{\tau \leq 0\}}^{\mathrm{b}}\left(\mathbf{k}_{M \times \mathbb{R}}\right)$ consisting of sheaves with microsupport contained in the set $\{\tau \leq 0\}$. He first proves the useful result which asserts that this localized category is equivalent to the left orthogonal to $\mathrm{D}_{\{\tau \leq 0\}}^{\mathrm{b}}\left(\mathbf{k}_{M \times \mathbb{R}}\right)$ and that the convolution by the sheaf $\mathbf{k}_{\{t \geq 0\}}$ is a projector on this left orthogonal.

Let us introduce the notation $\mathrm{D}^{\mathrm{b}}\left(\mathbf{k}_{M}^{\gamma}\right):=\mathrm{D}^{\mathrm{b}}\left(\mathbf{k}_{M \times \mathbb{R}} ;\{\tau>0\}\right)$ and, for a closed subset $A \subset T^{*} M$, let us denote by $\mathrm{D}_{A}^{\mathrm{b}}\left(\mathbf{k}_{M}^{\gamma}\right)$ the full triangulated subcategory of $\mathrm{D}^{\mathrm{b}}\left(\mathbf{k}_{M}^{\gamma}\right)$ consisting of objects with microsupport contained in $\rho^{-1} A$.

The first result of Tamarkin is a separability theorem. If $A$ and $B$ are two compact subsets of $T^{*} M, F \in \mathrm{D}_{A}^{\mathrm{b}}\left(\mathbf{k}_{M}^{\gamma}\right), G \in \mathrm{D}_{B}^{\mathrm{b}}\left(\mathbf{k}_{M}^{\gamma}\right)$, and if $A \cap B=\emptyset$, then $\operatorname{Hom}_{\mathrm{D}^{\mathrm{b}}\left(\mathbf{k}_{M}^{\gamma}\right)}(F, G) \simeq 0$.

The second result of Tamarkin is a Hamiltonian isotopy invariance theorem, up to torsion, that is, after killing what he calls the torsion objects. An object $F \in \mathrm{D}^{\mathrm{b}}\left(\mathbf{k}_{M}^{\gamma}\right)$ is torsion if there exists $c \geq 0$ such that the natural map $F \rightarrow T_{c *}(F)$ is zero, $T_{c *}(F)$ denoting the image of $F$ by the translation $t \mapsto t+c$ in the $t$-variable. Let $I$ be an open interval of $\mathbb{R}$ containing $[0,1]$ and let $\Phi=\left\{\varphi_{s}\right\}_{s \in I}$ be a Hamiltonian isotopy (with $\varphi_{0}=\mathrm{id}$ ) such that there 
exists a compact set $C \subset T^{*} M$ satisfying $\left.\varphi_{s}\right|_{T^{*} M \backslash C}=\operatorname{id}_{T^{*} M \backslash C}$ for all $s \in I$. Tamarkin constructs a functor $\Psi: \mathrm{D}_{A}^{\mathrm{b}}\left(\mathbf{k}_{M}^{\gamma}\right) \rightarrow \mathrm{D}_{\varphi_{1}(A)}^{\mathrm{b}}\left(\mathbf{k}_{M}^{\gamma}\right)$ such that $\Psi(F)$ is isomorphic to $F$ modulo torsion, for any $F \in \mathrm{D}_{A}^{\mathrm{b}}\left(\mathbf{k}_{M}^{\gamma}\right)$.

From these two results he easily deduces that if $A, B \subset T^{*} M$ are compact sets and if there exist $F \in \mathrm{D}_{A}^{\mathrm{b}}\left(\mathbf{k}_{M}^{\gamma}\right), G \in \mathrm{D}_{B}^{\mathrm{b}}\left(\mathbf{k}_{M}^{\gamma}\right)$ such that the map $\operatorname{RHom}_{\mathrm{D}^{\mathrm{b}}\left(\mathbf{k}_{M}^{\gamma}\right)}(F, G) \rightarrow \mathrm{RHom}_{\mathrm{D}^{\mathrm{b}}\left(\mathbf{k}_{M}^{\gamma}\right)}\left(F, T_{c}(G)\right)$ is not zero for all $c \geq 0$, then the sets $A$ and $B$ are mutually non displaceable, that is, for any Hamiltonian isotopy $\Phi$ as above and any $s \in I, A \cap \varphi_{s}(B) \neq \emptyset$.

Let us describe the contents of this paper.

In Section 1 we recall some constructions and results of [5] on the microlocal theory of sheaves.

In Section 2 we recall the main theorem of [2] which allows one to quantize homogeneous Hamiltonian isotopies and we also give some geometrical tools relying homogeneous and non homogeneous symplectic geometry.

In Section 3 we study convolution of sheaves on a trivial vector bundle $E=M \times V$ over $M$ as well as the category $\mathrm{D}^{\mathrm{b}}\left(\mathbf{k}_{E} ; U_{\gamma}\right)$, the localization of the category $\mathrm{D}^{\mathrm{b}}\left(\mathbf{k}_{E}\right)$ on $U_{\gamma}=E \times V \times \operatorname{Int}\left(\gamma_{0}^{\circ}\right)$ where $\operatorname{Int}\left(\gamma_{0}^{\circ}\right)$ is the interior of the polar cone to a closed convex proper cone $\gamma_{0}$ in $V$. We prove in particular a separability theorem in this category.

In Section 4 we introduce the Tamarkin category $\mathrm{D}^{\mathrm{b}}\left(\mathbf{k}_{M}^{\gamma}\right)$, that is, the category $\mathrm{D}^{\mathrm{b}}\left(\mathbf{k}_{E} ; U_{\gamma}\right)$ for $E=M \times \mathbb{R}$ and $\gamma_{0}=\{t \geq 0\}$.

In Section 5 we make a systematic study of the category $\mathscr{N}_{\text {tor }}$ of torsion objects, proving that this category is triangulated and also proving that, under some hypothesis on the microsupport, an object is torsion if and only if its restriction to one point is torsion (Theorem 5.12).

Finally, in Section 6 we give a proof of the Hamiltonian isotopy invariance theorem of Tamarkin. The existence of the functor $\Psi$ mentioned above is now an easy consequence on the results of [2], and one checks that this functor induces a functor isomorphic to the identity functor modulo torsion. As already mentioned, Tamarkin's non displaceability theorem is an easy corollary of the preceding results.

Note that, for the purposes we have in mind, we do not need to consider the unbounded derived category $\mathrm{D}\left(\mathbf{k}_{M}\right)$, as did Tamarkin, but only its full triangulated category $\mathrm{D}^{\mathrm{lb}}\left(\mathbf{k}_{M}\right)$ consisting of locally bounded objects. Also note that our notations, as well as our proofs, may seriously differ from Tamarkin's ones. 
In a next future, motivated by the papers of Fukaya-Seidel-Smith [1] and Nadler [8], we plan to use the tools developed here to study sheaves associated with smooth Lagrangian manifolds.

Acknowledgment We have been very much stimulated by the interest of Claude Viterbo for the applications of sheaf theory to symplectic topology and it is a pleasure to thank him here. We also thank Masaki Kashiwara for many enlightning discussions.

\section{Microlocal theory of sheaves}

In this section, we recall some definitions and results from [5], following its notations with the exception of slight modifications. We consider a real manifold $M$ of class $C^{\infty}$.

\section{Some geometrical notions $([\underline{5}, \S 4.2, \S 6.2])$}

For a locally closed subset $A$ of $M$, one denotes by $\operatorname{Int}(A)$ its interior and by $\bar{A}$ its closure. One denotes by $\Delta_{M}$ or simply $\Delta$ the diagonal of $M \times M$.

One denotes by $\tau: T M \rightarrow M$ and $\pi: T^{*} M \rightarrow M$ the tangent and cotangent bundles to $M$. If $L \subset M$ is a (smooth) submanifold, we denote by $T_{L} M$ its normal bundle and $T_{L}^{*} M$ its conormal bundle. They are defined by the exact sequences

$$
\begin{aligned}
& 0 \rightarrow T L \rightarrow L \times_{M} T M \rightarrow T_{L} M \rightarrow 0, \\
& 0 \rightarrow T_{L}^{*} M \rightarrow L \times_{M} T^{*} M \rightarrow T^{*} L \rightarrow 0 .
\end{aligned}
$$

One identifies $M$ to $T_{M}^{*} M$, the zero-section of $T^{*} M$. One sets $\dot{T}^{*} M:=T^{*} M \backslash$ $T_{M}^{*} M$ and one denotes by $\dot{\pi}_{M}: \dot{T}^{*} M \rightarrow M$ the projection.

Let $f: M \rightarrow N$ be a morphism of real manifolds. To $f$ are associated the tangent morphisms

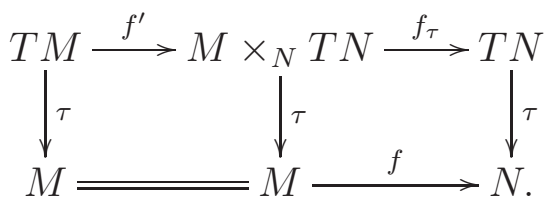


By duality, we deduce the diagram:

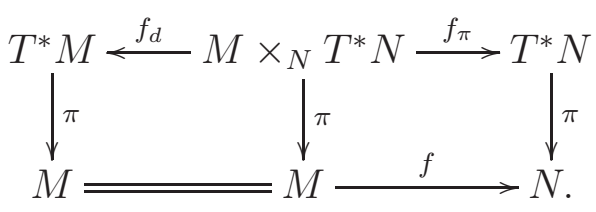

One sets

$$
T_{M}^{*} N:=\operatorname{Ker} f_{d}=f_{d}^{-1}\left(T_{M}^{*} M\right) .
$$

Note that, denoting by $\Gamma_{f}$ the graph of $f$ in $M \times N$, the projection $T^{*}(M \times$ $N) \rightarrow M \times T^{*} N$ identifies $T_{\Gamma_{f}}^{*}(M \times N)$ and $M \times_{N} T^{*} N$.

For two subsets $S_{1}, S_{2} \subset M$, their Whitney's normal cone, denoted $C\left(S_{1}, S_{2}\right)$, is the closed cone of $T M$ defined as follows. Let $(x)$ be a local coordinate system and let $(x ; v)$ denote the associated coordinate system on $T M$. Then

$$
\left\{\begin{array}{l}
\left(x_{0} ; v_{0}\right) \in C\left(S_{1}, S_{2}\right) \subset T M \text { if and only if there exists a se- } \\
\text { quence }\left\{\left(x_{n}, y_{n}, c_{n}\right)\right\}_{n} \subset S_{1} \times S_{2} \times \mathbb{R}^{+} \text {such that } x_{n} \stackrel{n}{\rightarrow} x_{0}, \\
y_{n} \stackrel{n}{\rightarrow} x_{0} \text { and } c_{n}\left(x_{n}-y_{n}\right) \stackrel{n}{\rightarrow} v_{0} .
\end{array}\right.
$$

For a subset $S$ of $M$ and a smooth closed submanifold $L$ of $M$, the Whitney's normal cone of $S$ along $L$, denoted $C_{L}(S)$, is the image in $T_{L} M$ of $C(L, S)$. If $L=\{p\}$, we write $C_{p}(S)$ instead of $C_{\{p\}}(S)$.

Now consider the homogeneous symplectic manifold $T^{*} M$ : it is endowed with the Liouville 1-form given in a local homogeneous symplectic coordinate system $(x ; \xi)$ on $T^{*} M$ by

$$
\alpha_{M}=\langle\xi, d x\rangle .
$$

The antipodal map $a_{M}$ is defined by:

$$
a_{M}: T^{*} M \rightarrow T^{*} M, \quad(x ; \xi) \mapsto(x ;-\xi) .
$$

If $A$ is a subset of $T^{*} M$, we denote by $A^{a}$ instead of $a_{M}(A)$ its image by the antipodal map.

We shall use the Hamiltonian isomorphism $H: T^{*}\left(T^{*} M\right) \stackrel{\sim}{\longrightarrow} T\left(T^{*} M\right)$ given in a local symplectic coordinate system $(x ; \xi)$ by

$$
H(\langle\lambda, d x\rangle+\langle\mu, d \xi\rangle)=-\left\langle\lambda, \partial_{\xi}\right\rangle+\left\langle\mu, \partial_{x}\right\rangle .
$$


Definition 1.1. (see [5, Def. 6.5.1]) A subset $S$ of $T^{*} M$ is co-isotropic (one also says involutive) at $p \in T^{*} M$ if for any $\theta \in T_{p}^{*} T^{*} M$ such that the Whitney normal cone $C_{p}(S, S)$ is contained in the hyperplane $\left\{v \in T T^{*} M ;\langle v, \theta\rangle=0\right\}$, one has $-H(\theta) \in C_{p}(S)$. A set $S$ is co-isotropic if it is so at each $p \in S$.

When $S$ is smooth, one recovers the usual notion.

\section{Microsupport}

We consider a commutative unital ring $\mathbf{k}$ of finite global dimension (e.g. $\mathbf{k}=\mathbb{Z})$. We denote by $\mathrm{D}\left(\mathbf{k}_{M}\right)$ (resp. $\mathrm{D}^{\mathrm{b}}\left(\mathbf{k}_{M}\right)$ ) the derived category (resp. bounded derived category) of sheaves of $\mathbf{k}$-modules on $M$.

Recall the definition of the microsupport (or singular support) $\operatorname{SS}(F)$ of a sheaf $F$.

Definition 1.2. (see [5, Def. 5.1.2]) Let $F \in \mathrm{D}^{\mathrm{b}}\left(\mathbf{k}_{M}\right)$ and let $p \in T^{*} M$. One says that $p \notin \mathrm{SS}(F)$ if there exists an open neighborhood $U$ of $p$ such that for any $x_{0} \in M$ and any real $C^{1}$-function $\varphi$ on $M$ defined in a neighborhood of $x_{0}$ satisfying $d \varphi\left(x_{0}\right) \in U$ and $\varphi\left(x_{0}\right)=0$, one has $\left(\mathrm{R}_{\{x ; \varphi(x) \geq 0\}}(F)\right)_{x_{0}} \simeq 0$.

In other words, $p \notin \mathrm{SS}(F)$ if the sheaf $F$ has no cohomology supported by "half-spaces" whose conormals are contained in a neighborhood of $p$.

- By its construction, the microsupport is closed and is $\mathbb{R}^{+}$-conic, that is, invariant by the action of $\mathbb{R}^{+}$on $T^{*} M$.

- $\operatorname{SS}(F) \cap T_{M}^{*} M=\pi_{M}(\mathrm{SS}(F))=\operatorname{Supp}(F)$.

- The microsupport satisfies the triangular inequality: if $F_{1} \rightarrow F_{2} \rightarrow$ $F_{3} \stackrel{+1}{\longrightarrow}$ is a distinguished triangle in $\mathrm{D}^{\mathrm{b}}\left(\mathbf{k}_{M}\right)$, then $\operatorname{SS}\left(F_{i}\right) \subset \operatorname{SS}\left(F_{j}\right) \cup$ $\mathrm{SS}\left(F_{k}\right)$ for all $i, j, k \in\{1,2,3\}$ with $j \neq k$.

Theorem 1.3. (see [5, Th. 6.5.4]) Let $F \in \mathrm{D}^{\mathrm{b}}\left(\mathbf{k}_{M}\right)$. Then its microsupport $\mathrm{SS}(F)$ is co-isotropic.

In the sequel, for a locally closed subset $Z$ in $M$, we denote by $\mathbf{k}_{Z}$ the constant sheaf with stalk $\mathbf{k}$ on $Z$, extended by 0 on $M \backslash Z$.

Example 1.4. (i) If $F$ is a non-zero local system on a connected manifold $M$, then $\mathrm{SS}(F)=T_{M}^{*} M$, the zero-section. 
(ii) If $N$ is a smooth closed submanifold of $M$ and $F=\mathbf{k}_{N}$, then $\operatorname{SS}(F)=$ $T_{N}^{*} M$, the conormal bundle to $N$ in $M$.

(iii) Let $\varphi$ be $C^{1}$-function with $d \varphi(x) \neq 0$ when $\varphi(x)=0$. Let $U=\{x \in$ $M ; \varphi(x)>0\}$ and let $Z=\{x \in M ; \varphi(x) \geq 0\}$. Then

$$
\begin{aligned}
& \mathrm{SS}\left(\mathbf{k}_{U}\right)=U \times_{M} T_{M}^{*} M \cup\{(x ; \lambda d \varphi(x)) ; \varphi(x)=0, \lambda \leq 0\}, \\
& \mathrm{SS}\left(\mathbf{k}_{Z}\right)=Z \times_{M} T_{M}^{*} M \cup\{(x ; \lambda d \varphi(x)) ; \varphi(x)=0, \lambda \geq 0\} .
\end{aligned}
$$

(iv) Let $\left(X, \mathscr{O}_{X}\right)$ be a complex manifold and let $\mathscr{M}$ be a coherent module over the ring $\mathscr{D}_{X}$ of holomorphic differential operators. (Hence, $\mathscr{M}$ represents a system of linear partial differential equations on $X$.) Denote by $F=\mathrm{R} \mathscr{H} \mathrm{om}_{\mathscr{D}_{X}}\left(\mathscr{M}, \mathscr{O}_{X}\right)$ the complex of holomorphic solutions of $\mathscr{M}$. Then $\mathrm{SS}(F)=\operatorname{char}(\mathscr{M})$, the characteristic variety of $\mathscr{M}$.

\section{Functorial operations (proper and non-characteristic cases)}

Let $M$ and $N$ be two real manifolds. We denote by $q_{i}(i=1,2)$ the $i$-th projection defined on $M \times N$ and by $p_{i}(i=1,2)$ the $i$-th projection defined on $T^{*}(M \times N) \simeq T^{*} M \times T^{*} N$.

Definition 1.5. Let $f: M \rightarrow N$ be a morphism of manifolds and let $\Lambda \subset$ $T^{*} N$ be a closed $\mathbb{R}^{+}$-conic subset. One says that $f$ is non-characteristic for $\Lambda$ (or else, $\Lambda$ is non-characteristic for $f$, or $f$ and $\Lambda$ are transversal) if

$$
f_{\pi}^{-1}(\Lambda) \cap T_{M}^{*} N \subset M \times_{N} T_{N}^{*} N .
$$

A morphism $f: M \rightarrow N$ is non-characteristic for a closed $\mathbb{R}^{+}$-conic subset $\Lambda$ of $T^{*} N$ if and only if $f_{d}: M \times_{N} T^{*} N \rightarrow T^{*} M$ is proper on $f_{\pi}^{-1}(\Lambda)$ and in this case $f_{d} f_{\pi}^{-1}(\Lambda)$ is closed and $\mathbb{R}^{+}$-conic in $T^{*} M$.

We denote by $\omega_{M}$ the dualizing complex on $M$. Recall that $\omega_{M}$ is isomorphic to the orientation sheaf shifted by the dimension. We also use the notation $\omega_{M / N}$ for the relative dualizing complex $\omega_{M} \otimes f^{-1} \omega_{N}^{\otimes-1}$. We have the duality functors

$$
\begin{aligned}
\mathrm{D}_{M}(\cdot) & =\mathrm{R} \mathscr{H} o m\left(\cdot, \omega_{M}\right), \\
\mathrm{D}_{M}^{\prime}(\cdot) & =\operatorname{R} \mathscr{H o m}\left(\cdot, \mathbf{k}_{M}\right) .
\end{aligned}
$$

Theorem 1.6. (See [5, § 5.4].) Let $f: M \rightarrow N$ be a morphism of manifolds, let $F \in \mathrm{D}^{\mathrm{b}}\left(\mathbf{k}_{M}\right)$ and let $G \in \mathrm{D}^{\mathrm{b}}\left(\mathbf{k}_{N}\right)$. 
(i) One has

$$
\begin{aligned}
& \mathrm{SS}(F \stackrel{\mathrm{L}}{\otimes} G) \subset \mathrm{SS}(F) \times \mathrm{SS}(G), \\
& \mathrm{SS}\left(\mathrm{R} \mathscr{H} \text { om }\left(q_{1}^{-1} F, q_{2}^{-1} G\right)\right) \subset \mathrm{SS}(F)^{a} \times \mathrm{SS}(G) .
\end{aligned}
$$

(ii) Assume that $f$ is proper on $\operatorname{Supp}(F)$. Then $\operatorname{SS}\left(\mathrm{R} f_{!} F\right) \subset f_{\pi} f_{d}^{-1} \operatorname{SS}(F)$.

(iii) Assume that $f$ is non-characteristic with respect to $\operatorname{SS}(G)$. Then the natural morphism $f^{-1} G \otimes \omega_{M / N} \rightarrow f^{!}(G)$ is an isomorphism. Moreover $\mathrm{SS}\left(f^{-1} G\right) \cup \mathrm{SS}\left(f^{!} G\right) \subset f_{d} f_{\pi}^{-1} \mathrm{SS}(G)$.

(iv) Assume that $f$ is smooth (that is, submersive). Then $\operatorname{SS}(F) \subset M \times_{N}$ $T^{*} N$ if and only if, for any $j \in \mathbb{Z}$, the sheaves $H^{j}(F)$ are locally constant on the fibers of $f$.

For the notion of a cohomologically constructible sheaf we refer to [5, $\S 3.4]$.

Corollary 1.7. Let $F_{1}, F_{2} \in \mathrm{D}^{\mathrm{b}}\left(\mathbf{k}_{M}\right)$.

(i) Assume that $\mathrm{SS}\left(F_{1}\right) \cap \mathrm{SS}\left(F_{2}\right)^{a} \subset T_{M}^{*} M$. Then

$$
\mathrm{SS}\left(F_{1} \stackrel{\mathrm{L}}{\otimes} F_{2}\right) \subset \mathrm{SS}\left(F_{1}\right)+\mathrm{SS}\left(F_{2}\right) .
$$

(ii) Assume that $\mathrm{SS}\left(F_{1}\right) \cap \mathrm{SS}\left(F_{2}\right) \subset T_{M}^{*} M$. Then

$$
\mathrm{SS}\left(\mathrm{R} \mathscr{H} o m\left(F_{1}, F_{2}\right)\right) \subset \mathrm{SS}\left(F_{1}\right)^{a}+\mathrm{SS}\left(F_{2}\right) .
$$

Moreover, assuming that $F_{1}$ is cohomologically constructible, the natural morphism $\mathrm{D}^{\prime} F_{1} \stackrel{\mathrm{L}}{\otimes} F_{2} \rightarrow \mathrm{R} \mathscr{H} o m\left(F_{1}, F_{2}\right)$ is an isomorphism.

The next result follows immediately from Theorem 1.6 (ii). It is a particular case of the microlocal Morse lemma (see [5, Cor. 5.4.19]), the classical theory corresponding to the constant sheaf $F=\mathbf{k}_{M}$.

Corollary 1.8. Let $F \in \mathrm{D}^{\mathrm{b}}\left(\mathbf{k}_{M}\right)$, let $\varphi: M \rightarrow \mathbb{R}$ be a function of class $C^{1}$ and assume that $\varphi$ is proper on $\operatorname{supp}(F)$. Let $a<b$ in $\mathbb{R}$ and assume that $d \varphi(x) \notin \mathrm{SS}(F)$ for $a \leq \varphi(x)<b$. Then the natural morphism $\mathrm{R} \Gamma\left(\varphi^{-1}(]-\infty, b[) ; F\right) \rightarrow \mathrm{R} \Gamma\left(\varphi^{-1}(]-\infty, a[) ; F\right)$ is an isomorphism. 
Corollary 1.9. Let $I$ be a contractible manifold and let $p: M \times I \rightarrow M$ be the projection. If $F \in \mathrm{D}^{\mathrm{b}}\left(\mathbf{k}_{M \times I}\right)$ satisfies $\mathrm{SS}(F) \subset T^{*} M \times T_{I}^{*} I$, then $F \simeq p^{-1} \mathrm{R} p_{*} F$.

Proof. It follows from Theorem 1.6 (iv) that the restriction $\left.F\right|_{\{x\} \times I}$ is locally constant for any $x \in M$. Then the result follows from [5, Prop. 2.7.8].

Corollary 1.10. Let $I$ be an open interval of $\mathbb{R}$ and let $q: M \times I \rightarrow I$ be the projection. Let $F \in \mathrm{D}^{\mathrm{b}}\left(\mathbf{k}_{M \times I}\right)$ such that $\mathrm{SS}(F) \cap\left(T_{M}^{*} M \times T^{*} I\right) \subset$ $T_{M \times I}^{*}(M \times I)$ and $q$ is proper on $\operatorname{Supp}(F)$. Then we have isomorphisms $\mathrm{R} \Gamma\left(M ; F_{s}\right) \simeq \mathrm{R} \Gamma\left(M ; F_{t}\right)$ for any $s, t \in I$.

Proof. It follows from Theorem 1.6 that $\mathrm{SS}\left(\mathrm{R} q_{*}(F)\right) \subset T_{I}^{*} I$. Hence, there exists $V \in \mathrm{D}^{\mathrm{b}}(\mathbf{k})$ and an isomorphism $\mathrm{R} q_{*}(F) \simeq V_{I}$. (Recall that $V_{I}=a_{I}^{-1} V$, where $a_{I} \rightarrow\{\mathrm{pt}\}$ is the projection and $V$ is identified to a sheaf on $\{\mathrm{pt}\}$.) Since we have $\mathrm{R} \Gamma\left(M ; F_{s}\right) \simeq\left(\mathrm{R} q_{*}(F)\right)_{s}$ the result follows.

\section{Kernels $([5, \S 3.6])$}

Notation 1.11. Let $M_{i}(i=1,2,3)$ be manifolds. For short, we write $M_{i j}:=M_{i} \times M_{j}(1 \leq i, j \leq 3)$ and $M_{123}=M_{1} \times M_{2} \times M_{3}$. We denote by $q_{i}$ the projection $M_{i j} \rightarrow M_{i}$ or the projection $M_{123} \rightarrow M_{i}$ and by $q_{i j}$ the projection $M_{123} \rightarrow M_{i j}$. Similarly, we denote by $p_{i}$ the projection $T^{*} M_{i j} \rightarrow T^{*} M_{i}$ or the projection $T^{*} M_{123} \rightarrow T^{*} M_{i}$ and by $p_{i j}$ the projection $T^{*} M_{123} \rightarrow T^{*} M_{i j}$. We also need to introduce the map $p_{12^{a}}$, the composition of $p_{12}$ and the antipodal map on $T^{*} M_{2}$.

Let $A \subset T^{*} M_{12}$ and $B \subset T^{*} M_{23}$. We set

$$
\begin{aligned}
& A \times_{T^{*} M_{2^{a}}} B=p_{12}^{-1}(A) \cap p_{2^{a} 3}^{-1}(B) \\
& A \stackrel{a}{\circ} B=p_{13}\left(A \times_{T^{*} M_{2^{a}}} B\right) \\
& =\left\{\left(x_{1}, x_{3} ; \xi_{1}, \xi_{3}\right) \in T^{*} M_{13} ; \text { there exists }\left(x_{2} ; \xi_{2}\right) \in T^{*} M_{2}\right. \text {, } \\
& \left.\left(x_{1}, x_{2} ; \xi_{1}, \xi_{2}\right) \in A,\left(x_{2}, x_{3} ;-\xi_{2}, \xi_{3}\right) \in B\right\} \text {. }
\end{aligned}
$$

We consider the operation of composition of kernels:

$$
\begin{aligned}
\circ: \mathrm{D}^{\mathrm{b}}\left(\mathbf{k}_{M_{12}}\right) \times \mathrm{D}^{\mathrm{b}}\left(\mathbf{k}_{M_{23}}\right) & \rightarrow \mathrm{D}^{\mathrm{b}}\left(\mathbf{k}_{M_{13}}\right) \\
\left(K_{1}, K_{2}\right) & \mapsto K_{1} \circ K_{2}:=\mathrm{R} q_{13 !}\left(q_{12}^{-1} K_{1} \stackrel{\mathrm{L}}{\otimes} q_{23}^{-1} K_{2}\right) .
\end{aligned}
$$


Let $A_{i}=\mathrm{SS}\left(K_{i}\right) \subset T^{*} M_{i, i+1}$ and assume that

$$
\left\{\begin{array}{c}
\left(\text { i) } q_{13} \text { is proper on } q_{12}^{-1} \operatorname{supp}\left(K_{1}\right) \cap q_{23}^{-1} \operatorname{supp}\left(K_{2}\right),\right. \\
\text { (ii) } p_{12}^{-1} A_{1} \cap p_{2^{a} 3}^{-1} A_{2} \cap\left(T_{M_{1}}^{*} M_{1} \times T^{*} M_{2} \times T_{M_{3}}^{*} M_{3}\right) \\
\subset T_{M_{1} \times M_{2} \times M_{3}}^{*}\left(M_{1} \times M_{2} \times M_{3}\right) .
\end{array}\right.
$$

It follows from Theorem 1.6 that under the assumption (8) we have:

$$
\mathrm{SS}\left(K_{1} \circ K_{2}\right) \subset A_{1} \stackrel{a}{\circ} A_{2}
$$

\section{Characteristic inverse images}

Theorem [1.6 treats the easy cases of external tensor product or external Hom, non-characteristic inverse images or proper direct image. In order to treat more general cases we introduce some additional geometrical notions.

Let $\Lambda$ be a smooth Lagrangian submanifold of $T^{*} M$. The Hamiltonian isomorphism defines an isomorphism

$$
T^{*} \Lambda \simeq T_{\Lambda} T^{*} M
$$

Let $j: L \hookrightarrow M$ be the embedding of a smooth submanifold $L$ of $M$. The Liouville form defines an embedding

$$
T^{*} L \hookrightarrow T^{*} T_{L}^{*} M \simeq T_{T_{L}^{*} M} T^{*} M
$$

Now consider a morphism of manifolds $f: M \rightarrow N$ and let us identify $M$ to the graph of $f$ in $M \times N$. For a subset $B \subset T^{*} N$ one sets:

$$
f^{\sharp}(B)=T^{*} M \cap C_{T_{M}^{*}(M \times N)}\left(T_{M}^{*} M \times B\right) .
$$

In local symplectic coordinate systems $(x ; \xi)$ on $M$ and $(y ; \eta)$ on $N$ one has

$$
\left\{\begin{array}{l}
\left(x_{0} ; \xi_{0}\right) \in f^{\sharp}(B) \text { if and only if there exist sequences } \\
\left\{x_{n}\right\}_{n} \subset M \text { and }\left\{\left(y_{n} ; \eta_{n}\right)\right\}_{n} \subset B \text { such that } \\
x_{n} \rightarrow x_{0},{ }^{t} f^{\prime}\left(x_{n}\right) \cdot \eta_{n} \stackrel{n}{\rightarrow} \xi_{0} \text { and }\left|y_{n}-f\left(x_{n}\right)\right| \cdot\left|\eta_{n}\right| \stackrel{n}{\rightarrow} 0 .
\end{array}\right.
$$

For two closed $\mathbb{R}^{+}$-conic subsets $A$ and $B$ of $T^{*} M$ one sets

$$
A \widehat{+} B=T^{*} M \cap C\left(A, B^{a}\right) .
$$


Here, $C\left(A, B^{a}\right)$ is considered as a subset of $T^{*} T^{*} M$ via the Hamiltonian isomorphism and $T^{*} M$ is embedded into $T^{*} T^{*} M$ via the Liouville form $\alpha_{M}$. In a local coordinate system, one has

$$
\left\{\begin{array}{l}
\left(z_{0} ; \zeta_{0}\right) \in A \widehat{+} B \text { if and only if there exist se- } \\
\text { quences }\left\{\left(x_{n} ; \xi_{n}\right)\right\}_{n} \text { in } A \text { and }\left\{\left(y_{n} ; \eta_{n}\right)\right\}_{n} \text { in } B \text { such } \\
\text { that } x_{n} \stackrel{n}{\rightarrow} z_{0}, y_{n} \stackrel{n}{\rightarrow} z_{0}, \xi_{n}+\eta_{n} \stackrel{n}{\rightarrow} \zeta_{0} \text { and } \\
\left|x_{n}-y_{n}\right| \cdot\left|\xi_{n}\right| \stackrel{n}{\rightarrow} 0 .
\end{array}\right.
$$

Theorem 1.12. (See [5, Cor. 6.4.4, 6.4.5].) Let $F_{1}, F_{2} \in \mathrm{D}^{\mathrm{b}}\left(\mathbf{k}_{M}\right)$ and let $G \in \mathrm{D}^{\mathrm{b}}\left(\mathbf{k}_{N}\right)$. Then

$$
\begin{aligned}
& \mathrm{SS}\left(F_{1} \otimes F_{2}\right) \subset \mathrm{SS}\left(F_{1}\right) \widehat{+} \mathrm{SS}\left(F_{2}\right), \\
& \mathrm{SS}\left(\mathrm{R} \mathscr{H} \circ m\left(F_{1}, F_{2}\right)\right) \subset \mathrm{SS}\left(F_{2}\right) \widehat{+} \mathrm{SS}\left(F_{1}\right)^{a}, \\
& \mathrm{SS}\left(f^{-1} G\right) \cup \mathrm{SS}\left(f^{!} G\right) \subset f^{\sharp}(\mathrm{SS}(G)) .
\end{aligned}
$$

\section{Non proper direct images}

We shall also need a direct image theorem in a non proper case.

Consider a constant linear map $u$ of trivial vector bundles over $M$, that is, we assume that $E_{i}=M \times V_{i}(i=1,2)$ and $u: V_{1} \rightarrow V_{2}$ is a linear map. The map $u$ defines the maps described by the diagram

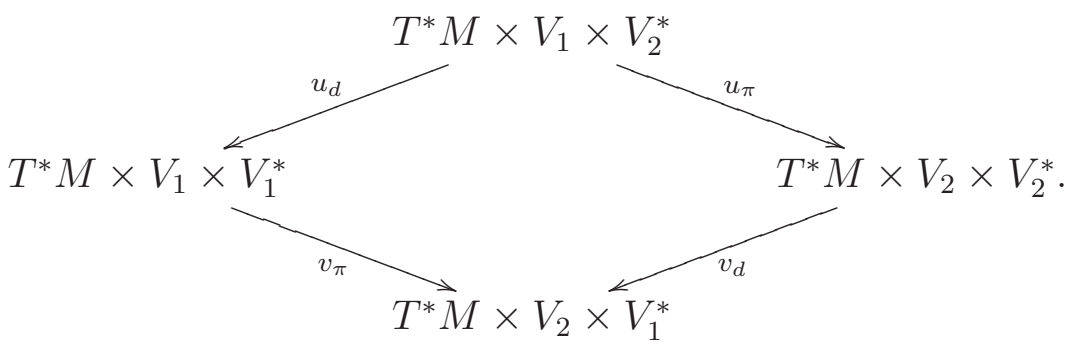

Note that for a subset $A$ of $T^{*} E_{1}$ we have

$$
u_{\pi}\left(u_{d}^{-1}(A)\right)=v_{d}^{-1}\left(v_{\pi}(A)\right) .
$$

Notation 1.13. Let $u: E_{1} \rightarrow E_{2}$ be a constant linear map of trivial vector bundles over $M$ and let $A \subset T^{*} E_{1}$ be a closed subset. We set

$$
u_{\sharp}(A)=v_{d}^{-1}\left(\overline{v_{\pi}(A)}\right) .
$$


In Lemmas 1.14 and 1.15 below we use the notations $\bigoplus_{n} G_{n}$ and $\prod_{n} G_{n}$ for a family $\left\{G_{n}\right\}_{n \in \mathbb{N}}$ in $\mathrm{D}^{\mathrm{b}}\left(\mathbf{k}_{M}\right)$. We define it as follows. Let $p: M \times \mathbb{N} \rightarrow$ $M$ be the projection. Then we have a unique $G \in \mathrm{D}^{\mathrm{b}}\left(\mathbf{k}_{M \times \mathbb{N}}\right)$ such that $\left.G\right|_{M \times\{n\}} \simeq G_{n}$, for all $n$, and we set $\bigoplus_{n} G_{n}:=\operatorname{R} p_{!} G$ and $\prod_{n} G_{n}:=\operatorname{R} p_{*} G$.

Lemma 1.14. Let $M$ be a manifolds and let $\left\{U_{n}\right\}_{n \in \mathbb{N}}$ be an increasing sequence of open subsets of $M$ such that $M=\bigcup_{n} U_{n}$. Then, for any $F \in$ $\mathrm{D}^{\mathrm{b}}\left(\mathbf{k}_{M}\right)$, we have the distinguished triangles

$$
\bigoplus_{n} F_{U_{n}} \stackrel{\mathrm{id}-s_{1}}{\longrightarrow} \bigoplus_{n} F_{U_{n}} \rightarrow F \stackrel{+1}{\longrightarrow}, \quad F \rightarrow \prod_{n} \mathrm{R} \Gamma_{U_{n}}(F) \stackrel{\mathrm{id}-s_{2}}{\longrightarrow} \prod_{n} \mathrm{R} \Gamma_{U_{n}}(F) \stackrel{+1}{\longrightarrow},
$$

where $s_{1}$ is the sum of the natural morphisms $F_{U_{n}} \rightarrow F_{U_{n+1}}$ and $s_{2}$ the product of the natural morphisms $\mathrm{R}_{U_{n+1}}(F) \rightarrow \mathrm{R} \Gamma_{U_{n}}(F)$ for $n \geq 0$ and the zero morphism for $n=-1$.

Proof. These triangles arise from similar exact sequences of sheaves when $F$ is a flabby sheaf. The exactness can be checked easily on the stalks in the first case and on sections over any open subset in the second case.

Lemma 1.15. Let $f: M \rightarrow N$ be a morphism of manifolds and let $\left\{U_{n}\right\}_{n \in \mathbb{N}}$ be an increasing sequence of open subsets of $M$ such that $M=\bigcup_{n} U_{n}$. Then, for any $F \in \mathrm{D}^{\mathrm{b}}\left(\mathbf{k}_{M}\right)$, we have

$$
\mathrm{SS}\left(\mathrm{R} f_{!} F\right) \subset \overline{\bigcup_{n} \operatorname{SS}\left(\mathrm{R} f_{!}\left(F_{U_{n}}\right)\right)}, \quad \mathrm{SS}\left(\mathrm{R} f_{*} F\right) \subset \overline{\bigcup_{n} \operatorname{SS}\left(\mathrm{R} f_{*} \mathrm{R} \Gamma_{U_{n}}(F)\right)} .
$$

Proof. We can check, similarly as in [5, Exe. V.7], that for any family $\left\{G_{n}\right\}_{n \in \mathbb{N}}$ in $\mathrm{D}^{\mathrm{b}}\left(\mathbf{k}_{N}\right)$ we have $\operatorname{SS}\left(\bigoplus_{n} G_{n}\right) \cup \operatorname{SS}\left(\prod_{n} G_{n}\right) \subset \overline{\bigcup_{n} \operatorname{SS}\left(G_{n}\right)}$. Then the result follows from Lemma 1.14 and the fact that $\mathrm{R} f$ ! commutes with $\oplus$ and $\mathrm{R} f_{*}$ with $\prod$.

The following result is due to Tamarkin [12, Lem. 3.3] but our proof is completely different.

Theorem 1.16. Let $u: E_{1} \rightarrow E_{2}$ be a constant linear map of trivial vector bundles over $M$ and let $F \in \mathrm{D}^{\mathrm{b}}\left(\mathbf{k}_{E_{1}}\right)$. Then $\mathrm{SS}\left(\mathrm{R} u_{!} F\right) \subset u_{\sharp}(\mathrm{SS}(F))$. The same estimate holds with $\mathrm{R} u_{!} F$ replaced with $\mathrm{R} u_{*} F$. 
Proof. (i) By decomposing $u$ by its graph, one is reduced to prove the result for an immersion and for a projection. Since the case of an immersion is obvious, we restrict ourselves to the case where $E=M \times V$ and $u: E \rightarrow M$ is the projection. Moreover the result is local on $M$ and we may assume that $M$ is an open subset in a vector space $W$.

(ii) We consider $\left(x_{0} ; \xi_{0}\right) \in T^{*} M \simeq M \times W^{*}$ such that $\left(x_{0} ; \xi_{0}\right) \notin u_{\sharp}(\operatorname{SS}(F))$. We will prove that $\left(x_{0} ; \xi_{0}\right) \notin \mathrm{SS}\left(\mathrm{R} u_{1} F\right) \cup \mathrm{SS}\left(\mathrm{R} u_{*} F\right)$. If $\xi_{0}=0$, then $\left.F\right|_{U \times V} \simeq$ 0 for some neighborhood $U$ of $x_{0}$ and the result follows easily. Hence we assume that $\xi_{0} \neq 0$. Up to shrinking $M$ we may find an open cone $C \subset$ $W^{*} \times V^{*}$ such that $\left(\xi_{0}, 0\right) \in C$ and $\operatorname{SS}(F) \cap((M \times V) \times C)=\emptyset$.

(iii) We choose an open convex cone $\gamma \subset W \times V$ such that $\bar{\gamma} \cap(\{0\} \times V)=$ $\{(0,0)\}$ and $\gamma^{\circ} \subset C$. We also choose two sequences of points $\left\{z_{n}\right\}_{n \in \mathbb{N}}$, resp. $\left\{z_{n}^{\prime}\right\}_{n \in \mathbb{N}}$, of $W \times V$ such that $W \times V$ is the increasing union of the cones $\gamma_{n}=z_{n}-\gamma$, resp. $\gamma_{n}^{\prime}=z_{n}^{\prime}+\gamma$. By Lemma 1.15 it is enough to show

$$
\left(\mathrm{SS}\left(\mathrm{R} u_{*} \mathrm{R} \Gamma_{\gamma_{n}} F\right) \cup \mathrm{SS}\left(\mathrm{R} u_{!}\left(F_{\gamma_{n}^{\prime}}\right)\right)\right) \cap\left(M \times\left(C \cap\left(W^{*} \times\{0\}\right)\right)=\emptyset .\right.
$$

(iv) By Lemma 3.16 below $\operatorname{SS}\left(\mathbf{k}_{\overline{\gamma_{n}}}\right) \subset(W \times V) \times(-C)$. Using $\mathrm{D}_{M}^{\prime}\left(\mathbf{k}_{\overline{\gamma_{n}}}\right) \simeq \mathbf{k}_{\gamma_{n}}$ we deduce $\mathrm{SS}\left(\mathbf{k}_{\gamma_{n}}\right) \subset(W \times V) \times C$. Similarly $\operatorname{SS}\left(\mathbf{k}_{\gamma_{n}^{\prime}}\right) \subset(W \times V) \times(-C)$. Since $\operatorname{SS}(F) \cap((M \times V) \times C)=\emptyset$, Corollary 1.7 gives

$$
\left(\mathrm{SS}\left(\mathrm{R}_{\gamma_{n}} F\right) \cup \mathrm{SS}\left(\left(F_{\gamma_{n}^{\prime}}\right)\right)\right) \cap((M \times V) \times C)=\emptyset .
$$

Since $\bar{\gamma} \cap(\{0\} \times V)=\{(0,0)\}$ the map $u: M \times V \rightarrow M$ is proper on all $\overline{\gamma_{n}}$ and $\overline{\gamma_{n}^{\prime}}$ and the result follows from Theorem 1.6 (ii).

For a trivial vector bundle $E=M \times V$ we denote by

$$
\widehat{\pi}_{E}: T^{*} E \rightarrow T^{*} M \times V^{*},
$$

or $\widehat{\pi}$ if there is no risk of confusion, the natural projection. We say that a subset of $T^{*} M \times V^{*}$ is a cone if it is stable by the multiplicative action of $\mathbb{R}^{+}$given by

$$
\lambda \cdot(x ; \xi, v)=(x ; \lambda \xi, \lambda v) .
$$

We will be mainly concerned with the case where $F \in \mathrm{D}^{\mathrm{b}}\left(\mathbf{k}_{E}\right)$ has a microsupport bounded by $\widehat{\pi}_{E}^{-1}(A)$ for some closed cone $A \subset T^{*} M \times V^{*}$. 
Let $u: E_{1}=M \times V_{1} \rightarrow E_{2}=M \times V_{2}$ be a constant linear map of trivial vector bundles over $M$ and denote by

$$
\widetilde{u}_{d}: T^{*} M \times V_{2}^{*} \rightarrow T^{*} M \times V_{1}^{*}
$$

the map associated with $u$.

Corollary 1.17. Let $u: E_{1} \rightarrow E_{2}$ be a constant linear map of trivial vector bundles over $M$ and let $F \in \mathrm{D}^{\mathrm{b}}\left(\mathbf{k}_{E_{1}}\right)$. Assume that $\operatorname{SS}(F) \subset \widehat{\pi}_{E_{1}}^{-1}\left(A_{1}\right)$ for a closed cone $A_{1} \subset T^{*} M \times V_{1}^{*}$. Then $\operatorname{SS}(\mathrm{R} u ! F) \subset \widehat{\pi}_{E_{2}}^{-1} \widetilde{u}_{d}^{-1}\left(A_{1}\right)$. The same estimate holds with $\mathrm{R} u_{!} F$ replaced with $\mathrm{R} u_{*} F$.

Proof. We have $v_{\pi}\left(\widehat{\pi}_{E_{1}}^{-1}\left(A_{1}\right)\right)=A_{1} \times V_{2}$ and this set is closed. We thus have

$$
\begin{aligned}
u_{\sharp}\left(\widehat{\pi}_{E_{1}}^{-1}\left(A_{1}\right)\right) & =v_{d}^{-1}\left(v_{\pi}\left(\widehat{\pi}_{E_{1}}^{-1}\left(A_{1}\right)\right)\right)=u_{\pi}\left(u_{d}^{-1}\left(A_{1} \times V_{1}\right)\right) \\
& =\widetilde{u}_{d}^{-1}\left(A_{1}\right) \times V_{2}=\widehat{\pi}_{E_{2}}^{-1} \widetilde{u}_{d}^{-1}\left(A_{1}\right) .
\end{aligned}
$$

\section{Localization}

Let $\mathscr{T}$ be a triangulated category, $\mathscr{N}$ a null system, that is, a full triangulated subcategory with the property that if one has an isomorphism $F \simeq G$ in $\mathscr{T}$ with $F \in \mathscr{N}$, then $G \in \mathscr{N}$. The localization $\mathscr{T} / \mathscr{N}$ is a well defined triangulated category (we skip the problem of universes). Its objects are those of $\mathscr{T}$ and a morphism $u: F_{1} \rightarrow F_{2}$ in $\mathscr{T}$ becomes an isomorphism in $\mathscr{T} / \mathscr{N}$ if, after embedding this morphism in a distinguished triangle $F_{1} \rightarrow$ $F_{2} \rightarrow F_{3} \stackrel{+1}{\longrightarrow}$, one has $F_{3} \in \mathscr{N}$.

Recall that the left orthogonal $\mathscr{N}^{\perp, l}$ of $\mathscr{N}$ is the full triangulated subcategory of $\mathscr{T}$ defined by:

$$
\mathscr{N}^{\perp, l}=\left\{F \in \mathscr{T} ; \operatorname{Hom}_{\mathscr{T}}(F, G) \simeq 0 \text { for all } G \in \mathscr{N}\right\} .
$$

By classical results (see e.g., [6, Exe. 10.15]), if the embedding $\mathscr{N}^{\perp, l} \hookrightarrow$ $\mathscr{T}$ admits a left adjoint, or equivalently, if for any $F \in \mathscr{T}$, there exists a distinguished triangle $F^{\prime} \rightarrow F \rightarrow F^{\prime \prime} \stackrel{+1}{\longrightarrow}$ with $F^{\prime} \in \mathscr{N}^{\perp, l}$ and $F^{\prime \prime} \in \mathscr{N}$, then there is an equivalence $\mathscr{N}^{\perp, l} \simeq \mathscr{T} / \mathscr{N}$.

Of course, there are similar results with the right orthogonal $\mathscr{N}^{\perp, r}$. 
Now let $U$ be a subset of $T^{*} M$ and set $Z=T^{*} M \backslash U$. The full subcategory $\mathrm{D}_{Z}^{\mathrm{b}}\left(\mathbf{k}_{M}\right)$ of $\mathrm{D}^{\mathrm{b}}\left(\mathbf{k}_{M}\right)$ consisting of sheaves $F$ such that $\mathrm{SS}(F) \subset Z$ is a null system. One sets

$$
\mathrm{D}^{\mathrm{b}}\left(\mathbf{k}_{M} ; U\right):=\mathrm{D}^{\mathrm{b}}\left(\mathbf{k}_{M}\right) / \mathrm{D}_{Z}^{\mathrm{b}}\left(\mathbf{k}_{M}\right),
$$

the localization of $\mathrm{D}^{\mathrm{b}}\left(\mathbf{k}_{M}\right)$ by $\mathrm{D}_{Z}^{\mathrm{b}}\left(\mathbf{k}_{M}\right)$. Hence, the objects of $\mathrm{D}^{\mathrm{b}}\left(\mathbf{k}_{M} ; U\right)$ are those of $\mathrm{D}^{\mathrm{b}}\left(\mathbf{k}_{M}\right)$ but a morphism $u: F_{1} \rightarrow F_{2}$ in $\mathrm{D}^{\mathrm{b}}\left(\mathbf{k}_{M}\right)$ becomes an isomorphism in $\mathrm{D}^{\mathrm{b}}\left(\mathbf{k}_{M} ; U\right)$ if, after embedding this morphism in a distinguished triangle $F_{1} \rightarrow F_{2} \rightarrow F_{3} \stackrel{+1}{\rightarrow}$, one has $\mathrm{SS}\left(F_{3}\right) \cap U=\emptyset$.

For a closed subset $A$ of $U, \mathrm{D}_{A}^{\mathrm{b}}\left(\mathbf{k}_{M} ; U\right)$ denotes the full triangulated subcategory of $\mathrm{D}^{\mathrm{b}}\left(\mathbf{k}_{M} ; U\right)$ consisting of objects whose microsupports have an intersection with $U$ contained in $A$.

\section{Quantized symplectic isomorphisms ([5, §7.2])}

Consider two manifolds $M$ and $N$, two conic open subsets $U \subset T^{*} M$ and $V \subset T^{*} N$ and a homogeneous symplectic isomorphism $\chi$ :

$$
T^{*} N \supset V \underset{\chi}{\sim} U \subset T^{*} M
$$

Denote by $V^{a}$ the image of $V$ by the antipodal map $a_{N}$ on $T^{*} N$ and by $\Lambda$ the image of the graph of $\varphi$ by $\operatorname{id}_{U} \times a_{N}$. Hence $\Lambda$ is a conic Lagrangian submanifold of $U \times V^{a}$. A quantized contact transformation (a QCT, for short) above $\chi$ is a kernel $K \in \mathrm{D}^{\mathrm{b}}\left(\mathbf{k}_{M \times N}\right)$ such that $\mathrm{SS}(K) \cap\left(U \times V^{a}\right) \subset \Lambda$ and satisfying some technical properties that we do not recall here, so that the kernel $K$ induces an equivalence of categories

$$
K \circ \cdot: \mathrm{D}^{\mathrm{b}}\left(\mathbf{k}_{N} ; V\right) \stackrel{\sim}{\longrightarrow} \mathrm{D}^{\mathrm{b}}\left(\mathbf{k}_{M} ; U\right) .
$$

Given $\chi$ and $q \in V, p=\chi(q) \in U$, there exists such a QCT after replacing $U$ and $V$ by sufficiently small neighborhoods of $p$ and $q$.

\section{Simple sheaves $([\underline{5}, \S 7.5])$}

Let $\Lambda \subset \dot{T}^{*} M$ be a locally closed conic Lagrangian submanifold and let $p \in \Lambda$. Simple sheaves along $\Lambda$ at $p$ are defined in [5, Def. 7.5.4].

When $\Lambda$ is the conormal bundle to a submanifold $N \subset M$, that is, when the projection $\left.\pi_{M}\right|_{\Lambda}: \Lambda \rightarrow M$ has constant rank, then an object $F \in \mathrm{D}^{\mathrm{b}}\left(\mathbf{k}_{M}\right)$ is simple along $\Lambda$ at $p$ if $F \simeq \mathbf{k}_{N}[d]$ in $\mathrm{D}^{\mathrm{b}}\left(\mathbf{k}_{M} ; p\right)$ for some shift $d \in \mathbb{Z}$. 
If $\operatorname{SS}(F)$ is contained in $\Lambda$ on a neighborhood of $\Lambda, \Lambda$ is connected and $F$ is simple at some point of $\Lambda$, then $F$ is simple at every point of $\Lambda$.

The functor $\mu$ hom $([5, \S 4.4, \S 7.2])$

The functor of microlocalization along a submanifold has been introduced by Mikio Sato in the 70's and has been at the origin of what is now called "microlocal analysis". A variant of this functor, the bifunctor

$$
\text { uhom: } \mathrm{D}^{\mathrm{b}}\left(\mathbf{k}_{M}\right)^{\mathrm{op}} \times \mathrm{D}^{\mathrm{b}}\left(\mathbf{k}_{M}\right) \rightarrow \mathrm{D}^{\mathrm{b}}\left(\mathbf{k}_{T^{*} M}\right)
$$

has been constructed in [5]. Let us only recall the properties of this functor that we shall use. For $F, G \in \mathrm{D}^{\mathrm{b}}\left(\mathbf{k}_{M}\right)$, with $F$ cohomologically constructible, we have

$$
\begin{aligned}
\mathrm{R} \pi_{M *} \mu h o m(F, G) & \simeq \mathrm{R} \mathscr{H o m}(F, G), \\
\mathrm{R} \pi_{M !} \mu h o m(F, G) & \simeq \mathrm{D}_{M}^{\prime}(F) \otimes G
\end{aligned}
$$

and we deduce the distinguished triangle

$$
\mathrm{D}_{M}^{\prime}(F) \stackrel{\mathrm{L}}{\otimes} G \rightarrow \mathrm{R} \mathscr{H} o m(F, G) \rightarrow \mathrm{R} \dot{\pi}_{M *}\left(\left.\mu h o m(F, G)\right|_{\dot{T}^{*} M}\right) \stackrel{+1}{\longrightarrow} .
$$

Let $\Lambda \subset \dot{T}^{*} M$ be a locally closed smooth conic Lagrangian submanifold and let $F \in \mathrm{D}^{\mathrm{b}}\left(\mathbf{k}_{M}\right)$ be simple along $\Lambda$. Then

$$
\left.\mu h o m(F, F)\right|_{\Lambda} \simeq \mathbf{k}_{\Lambda} .
$$

\section{Quantization of Hamiltonian isotopies}

In this section, we recall the main theorem of [2].

We first recall some notions of symplectic geometry. Let $\mathfrak{X}$ be a symplectic manifold with symplectic form $\omega$. We denote by $\mathfrak{X}^{a}$ the same manifold endowed with the symplectic form $-\omega$. The symplectic structure induces the Hamiltonian isomorphism $\mathbf{h}: T \mathfrak{X} \stackrel{\sim}{\longrightarrow} T^{*} \mathfrak{X}$ by $\mathbf{h}(v)=\iota_{v}(\omega)$, where $\iota_{v}$ denotes the contraction with $v$ (in case $\mathfrak{X}$ is a cotangent bundle we have $\mathbf{h}=-H^{-1}$, where $H$ is used in Definition 1.1). To a vector field $v$ on $\mathfrak{X}$ we associate in this way a 1 -form $\mathbf{h}(v)$ on $\mathfrak{X}$. For a $C^{\infty}$-function $f: \mathfrak{X} \rightarrow \mathbb{R}$, the Hamiltonian vector field of $f$ is by definition $H_{f}:=-\mathbf{h}^{-1}(d f)$. 
A vector field $v$ is called symplectic if its flow preserves $\omega$. This is equivalent to $\mathscr{L}_{v}(\omega)=0$ where $\mathscr{L}_{v}$ denotes the Lie derivative of $v$. By Cartan's formula $\left(\mathscr{L}_{v}=d \iota_{v}+\iota_{v} d\right)$ this is again equivalent to $d(\mathbf{h}(v))=0$ (recall that $d \omega=0)$. The vector field $v$ is called Hamiltonian if $\mathbf{h}(v)$ is exact, or equivalently $v=H_{f}$ for some function $f$ on $\mathfrak{X}$.

Let $I$ be an open interval of $\mathbb{R}$ containing the origin and let $\Phi: \mathfrak{X} \times I \rightarrow \mathfrak{X}$ be a map such that $\varphi_{s}:=\Phi(\cdot, s): \mathfrak{X} \rightarrow \mathfrak{X}$ is a symplectic isomorphism for each $s \in I$ and is the identity for $s=0$. The map $\Phi$ induces a time dependent vector field on $\mathfrak{X}$

$$
v_{\Phi}:=\frac{\partial \Phi}{\partial s}: \mathfrak{X} \times I \rightarrow T \mathfrak{X}
$$

The "time dependent" 1 -form $\beta=\mathbf{h}\left(v_{\Phi}\right): \mathfrak{X} \times I \rightarrow T^{*} \mathfrak{X}$ satisfies $d\left(\beta_{s}\right)=0$ for any $s \in I$. The map $\Phi$ is called a Hamiltonian isotopy if $v_{\Phi, s}$ is Hamiltonian, that is, if $\beta_{s}$ is exact, for any $s$. In this case we can write $\beta_{s}=-d\left(f_{s}\right)$ for some $C^{\infty}$-function $f: \mathfrak{X} \times I \rightarrow \mathbb{R}$. Hence we have

$$
\frac{\partial \Phi}{\partial s}=H_{f_{s}} .
$$

The fact that the isotopy $\Phi$ is Hamiltonian can be interpreted as a geometric property of its graph as follows. For a given $s \in I$ we let $\Lambda_{s}$ be the graph of $\varphi_{s}^{-1}$ and we let $\Lambda^{\prime}$ be the family of $\Lambda_{s}^{\prime}$ 's:

$$
\begin{aligned}
& \Lambda_{s}=\left\{\left(\varphi_{s}(v), v\right) ; v \in \mathfrak{X}^{a}\right\} \subset \mathfrak{X} \times \mathfrak{X}^{a}, \\
& \Lambda^{\prime}=\left\{\left(\varphi_{s}(v), v, s\right) ; v \in \mathfrak{X}^{a}, s \in I\right\} \subset \mathfrak{X} \times \mathfrak{X}^{a} \times I .
\end{aligned}
$$

Thus $\Lambda_{s}$ is a Lagrangian submanifold of $\mathfrak{X} \times \mathfrak{X}^{a}$. Now we can see that $\Phi$ is a Hamiltonian isotopy if and only if there exists a Lagrangian submanifold $\Lambda \subset \mathfrak{X} \times \mathfrak{X}^{a} \times T^{*} I$ such that, for any $s \in I$,

$$
\Lambda_{s}=\Lambda \circ T_{s}^{*} I \text {. }
$$

(Here, the notation $\bullet \circ \cdot$ is a slight generalization of (6) to the case where the symplectic manifolds are no more cotangent bundles.) In this case $\Lambda$ is written

$$
\Lambda=\{(\Phi(v, s), v, s,-f(\Phi(v, s), s)) ; v \in \mathfrak{X}, s \in I\},
$$

where the function $f: \mathfrak{X} \times I \rightarrow \mathbb{R}$ is defined up to addition of a function depending on $s$ by $v_{\Phi, s}=H_{f_{s}}$. 


\section{Homogeneous case}

Let us come back to the case $\mathfrak{X}=\dot{T}^{*} M$ and consider $\Phi: \dot{T}^{*} M \times I \rightarrow \dot{T}^{*} M$ such that

$$
\left\{\begin{array}{l}
\varphi_{s} \text { is a homogeneous symplectic isomorphism for each } s \in I, \\
\varphi_{0}=\mathrm{id}_{\dot{T}^{*} M} .
\end{array}\right.
$$

In this case $\Phi$ is a Hamiltonian isotopy and there exists a unique homogeneous function $f$ such that $v_{\Phi, s}=H_{f_{s}}$. It is given by

$$
f=\left\langle\alpha, v_{\Phi}\right\rangle: \dot{T}^{*} M \times I \rightarrow \mathbb{R} .
$$

Since $f$ is homogeneous of degree 1 in the fibers of $\dot{T}^{*} M$, the Lagrangian submanifold $\Lambda$ of $\dot{T}^{*} M \times \dot{T}^{*} M \times T^{*} I$ associated to $f$ in (26) is $\mathbb{R}^{+}$-conic.

We say that $F \in \mathrm{D}\left(\mathbf{k}_{M}\right)$ is locally bounded if for any relatively compact open subset $U \subset M$ we have $\left.F\right|_{U} \in \mathrm{D}^{\mathrm{b}}\left(\mathbf{k}_{U}\right)$. We denote by $\mathrm{D}^{\mathrm{lb}}\left(\mathbf{k}_{M}\right)$ the full subcategory of $\mathrm{D}\left(\mathbf{k}_{M}\right)$ consisting of locally bounded objects.

Theorem 2.1. ([2, Th 4.3].) Consider a homogeneous Hamiltonian isotopy $\Phi$ satisfying the hypotheses (27). Let us consider the following conditions on $K \in \mathrm{D}^{\mathrm{lb}}\left(\mathbf{k}_{M \times M \times I}\right)$ :

(a) $\operatorname{SS}(K) \subset \Lambda \cup T_{M \times M \times I}^{*}(M \times M \times I)$,

(b) $K_{0} \simeq \mathbf{k}_{\Delta}$,

(c) both projections $\operatorname{Supp}(K) \rightrightarrows M \times I$ are proper,

(d) $K_{s} \circ K_{s}^{-1} \simeq K_{s}^{-1} \circ K_{s} \simeq \mathbf{k}_{\Delta}$, where $K_{s}^{-1}=v^{-1} \mathrm{R} \mathscr{H} \circ m\left(K_{s}, \omega_{M} \otimes \mathbf{k}_{M}\right)$ and $v(x, y)=(y, x)$.

Then we have

(i) The conditions (国) and (b) imply the other two conditions (ㅁ) and (d) .

(ii) There exists $K$ satisfying (国) -(d) .

(iii) Moreover such a $K$ satisfying the conditions (国)-(䏓) is unique up to a unique isomorphism.

We shall call $K$ the quantization of $\Phi$ on $I$, or the quantization of the family $\left\{\varphi_{s}\right\}_{s \in I}$. 


\section{Non homogeneous case}

Theorem 2.1 is concerned with homogeneous Hamiltonian isotopies. The next result will allow us to adapt it to non homogeneous cases. Let $\Phi: T^{*} M \times I \rightarrow$ $T^{*} M$ be a Hamiltonian isotopy and assume

$$
\left\{\begin{array}{l}
\text { there exists a compact set } C \subset T^{*} M \text { such that } \\
\left.\varphi_{s}\right|_{T^{*} M \backslash C} \text { is the identity for all } s \in I .
\end{array}\right.
$$

We denote by $T_{\{\tau>0\}}^{*}(M \times \mathbb{R})$ the open subset $\{\tau>0\}$ of $T^{*}(M \times \mathbb{R})$ and we define the map

$$
\rho: T_{\{\tau>0\}}^{*}(M \times \mathbb{R}) \rightarrow T^{*} M, \quad(x, t ; \xi, \tau) \mapsto(x ; \xi / \tau) .
$$

Proposition 2.2. ([2, Prop. A.6].) There exist a homogeneous Hamiltonian isotopy $\widetilde{\Phi}: \dot{T}^{*}(M \times \mathbb{R}) \times I \rightarrow \dot{T}^{*}(M \times \mathbb{R})$ and $C^{\infty}$-functions $u: T^{*} M \times I \rightarrow \mathbb{R}$ and $v: I \rightarrow \mathbb{R}$ such that the following diagram commutes:

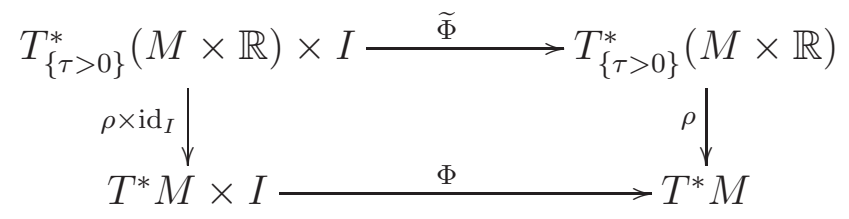

and

$$
\begin{aligned}
& \widetilde{\Phi}((x ; \xi),(t ; \tau), s)=\left(\left(x^{\prime} ; \xi^{\prime}\right),(t+u(x ; \xi / \tau, s) ; \tau)\right), \\
& \widetilde{\Phi}((x ; \xi),(t ; 0), s)=((x ; \xi),(t+v(s) ; 0)),
\end{aligned}
$$

where $\left(x^{\prime} ; \xi^{\prime} / \tau\right)=\varphi_{s}(x ; \xi / \tau)$. Moreover we have $u(x ; \xi / \tau, s)=v(s)$ for $(x ; \xi / \tau) \notin C$.

\section{Convolution and localization}

Most of the ideas of this section are due to Tamarkin [12]. The reader will be aware that our notations do not follow Tamarkin's ones. We also give some proofs which may be rather different from Tamarkin's original ones.

In all this section, we consider a trivial vector bundle

$$
q: E=M \times V \rightarrow M
$$


and a trivial cone $\gamma=M \times \gamma_{0} \subset E$ such that

$\gamma_{0}$ is a closed convex proper cone of $V$ containing 0 and $\gamma_{0} \neq\{0\}$.

The polar cone $\gamma_{0}^{\circ} \subset V^{*}$ is the closed convex cone given by

$$
\gamma_{0}^{\circ}=\left\{\theta \in V^{*} ;\langle\theta, v\rangle \geq 0\right\} \text { for all } v \in \gamma_{0} .
$$

Many results could be generalized to general vector bundles and general proper convex cones, but in practice we shall use these results with $V=\mathbb{R}$ and $\gamma_{0}=\{t \in \mathbb{R} ; t \geq 0\}$. Recall that a subset in $T^{*} M \times V^{*}$ is a cone if it is invariant by the diagonal action of $\mathbb{R}^{+}$(see (17)).

Definition 3.1. A closed cone $A \subset T^{*} M \times V^{*}$ is called a strict $\gamma$-cone if $A \subset\left(T^{*} M \times \operatorname{Int} \gamma_{0}^{\circ}\right) \cup T_{M}^{*} M \times\{0\}$.

Example 3.2. Assume $V=\mathbb{R}$ and $M$ is open in $\mathbb{R}^{n}$. Denote by $(t ; \tau)$ the coordinates on $T^{*} \mathbb{R}$ and by $(x ; \xi)$ the coordinates on $T^{*} M$. Let $\gamma_{0}=\{t \in$ $\mathbb{R} ; t \geq 0\}$. Then a closed cone $A \subset T^{*} M \times V^{*}$ is a strict $\gamma$-cone if, for any compact subset $C \subset M$, there exists $a \in \mathbb{R}, a>0$ such that $\tau \geq a|\xi|$ for all $(x ; \xi, \tau) \in A \cap\left(\pi_{M}^{-1}(C) \times V^{*}\right)$.

Remark 3.3. If $f: N \rightarrow M$ is a morphism of manifolds and $A \subset T^{*} M \times V^{*}$ is a strict $\gamma$-cone, then $f \times \operatorname{id}_{V}: N \times V \rightarrow M \times V$ is non-characteristic for $\widehat{\pi}_{E}^{-1}(A)$ (where $\widehat{\pi}_{E}^{-1}$ is defined in (16)) .

In the sequel, we consider the maps

$$
\begin{gathered}
q_{1}, q_{2}, s: V \times V \rightarrow V, \\
q_{1}\left(v_{1}, v_{2}\right)=v_{1}, \quad q_{2}\left(v_{1}, v_{2}\right)=v_{2}, \quad s\left(v_{1}, v_{2}\right)=v_{1}+v_{2} .
\end{gathered}
$$

If there is no risk of confusion, we still denote by $q_{1}, q_{2}, s$ the associated maps $M \times V \times V \rightarrow M \times V$.

We denote by $\delta_{M}$ the diagonal embedding

$$
\delta_{M}: M \hookrightarrow M \times M
$$

and if there is no risk of confusion, we still denote by $\delta_{M}$ the associated map $M \times V \times V \hookrightarrow M \times M \times V \times V$, that is, the map $E \times_{M} E \hookrightarrow E \times E$.

The maps $s$ and $\delta_{M}$ give rise to the maps:

$$
\begin{aligned}
& T^{*}\left(E \times_{M} E\right) \stackrel{\left(\delta_{M}\right)_{d}}{\longleftarrow} M \times_{M \times M} T^{*}\left(E \times_{M} E\right) \stackrel{\left(\delta_{M}\right)_{\pi}}{\longrightarrow} T^{*}(E \times E), \\
& T^{*}\left(E \times_{M} E\right) \stackrel{s_{d}}{\longleftarrow} V \times_{V \times V} T^{*}\left(E \times_{M} E\right) \stackrel{s_{\pi}}{\longrightarrow} T^{*} E .
\end{aligned}
$$


On $T^{*} E$ we have the antipodal map $a$, but there is another involution associated with $a$ and the involution $(x, y) \mapsto(x,-y)$ on $E$. We denote by $\alpha$ the involution of $T^{*} E$

$$
\alpha:(x, y ; \xi, \eta) \mapsto(x,-y ;-\xi, \eta)
$$

and for a subset $A \subset T^{*} E$ we denote by $A^{\alpha}$ its image by this involution. We also denote by $\alpha$ the involution of $T^{*} M \times V^{*}$ defined by $(x ; \xi, \eta) \mapsto$ $(x ;-\xi, \eta)$. Hence for $A \subset T^{*} M \times V^{*}$ we have, using the notation (16), $\widehat{\pi}_{E}^{-1}\left(A^{\alpha}\right)=\widehat{\pi}_{E}^{-1}(A)^{\alpha}$.

\section{Convolution}

Recall the notations (10) and (15).

Notation 3.4. For two closed subsets $A$ and $B$ in $T^{*} E$, we set

$$
A \widehat{\star} B:=s_{\sharp} \delta_{M}^{\sharp}(A \times B) .
$$

In general, the calculation of $A \widehat{\star} B$ is difficult. In Lemmas 3.5 and 3.7 below we consider special situations in which this calculation is easy.

Lemma 3.5. Let $A^{\prime}$ and $B^{\prime}$ be two closed cones in $V^{*}$. Set $A=T^{*} M \times V \times A^{\prime}$ and $B=T^{*} M \times V \times B^{\prime}$. Then

$$
A \widehat{\star} B=A \cap B \text {. }
$$

Proof. Using the hypothesis on $A$ and $B$, it follows from (11) that

$$
\delta_{M}^{\sharp}(A \times B)=T^{*} M \times V \times V \times A^{\prime} \times B^{\prime} .
$$

Then the result follows from Corollary 1.17

Notation 3.6. Let $A$ and $B$ be two closed cones in $T^{*} M \times V^{*}$. We set

$$
\begin{array}{r}
\underset{M}{A+B}=\left\{(x ; \xi, \eta) \in T^{*} M \times V^{*} ; \text { there exist } \xi_{1}, \xi_{2} \in T_{x}^{*} M\right. \text { such } \\
\text { that } \left.\left(x ; \xi_{1}, \eta\right) \in A,\left(x ; \xi_{2}, \eta\right) \in B \text { and } \xi=\xi_{1}+\xi_{2}\right\} .
\end{array}
$$

Lemma 3.7. Consider two closed strict $\gamma$-cones $A$ and $B$ in $T^{*} M \times V^{*}$. Then $A+{ }_{M} B$ is also a strict $\gamma$-cone and $\widehat{\pi}_{E}^{-1}(A) \widehat{\star} \widehat{\pi}_{E}^{-1}(B)=\widehat{\pi}_{E}^{-1}(A+B)$.

In particular, if $A \cap B \subset T_{M}^{*} M \times\{0\}$, then

$$
\left(\widehat{\pi}_{E}^{-1}(A) \widehat{\star}\left(\widehat{\pi}_{E}^{-1}(B)\right)^{\alpha}\right) \cap\left(T_{M}^{*} M \times T^{*} V\right) \subset T_{E}^{*} E .
$$


Proof. The fact that $A+B$ is a strict $\gamma$-cone follows easily from the definition.

By Remark 3.3, $\widehat{\pi}_{E}^{-1}(A) \times \widehat{\pi}_{E}^{-1}(B)$ is non-characteristic for the inclusion $\delta_{M}: M \times V \times V \rightarrow M \times M \times V \times V$ and we may replace $\delta_{M}^{\sharp}$ by $\delta_{M, d} \delta_{M, \pi}^{-1}$ in (38). We find $\delta_{M}^{\sharp}\left(\widehat{\pi}_{E}^{-1}(A) \times\left(\widehat{\pi}_{E}^{-1}(B)\right)\right)=\widehat{\pi}_{M \times V \times V}^{-1}\left(C_{1}\right)$, where

$$
\begin{aligned}
C_{1}=\left\{\left(x ; \xi, \eta_{1}, \eta_{2}\right)\right. & \in T^{*} M \times V^{*} \times V^{*} ; \text { there exist } \xi_{1}, \xi_{2} \in T_{x}^{*} M \text { such } \\
& \text { that } \left.\left(x ; \xi_{1}, \eta_{1}\right) \in A,\left(x ; \xi_{2}, \eta_{2}\right) \in B \text { and } \xi=\xi_{1}+\xi_{2}\right\}
\end{aligned}
$$

and the result follows.

Using the notations (35), the convolution of sheaves is defined by:

Definition 3.8. For $F, G \in \mathrm{D}^{\mathrm{b}}\left(\mathbf{k}_{E}\right)$, we set

$$
\begin{aligned}
F \star G & :=\mathrm{R} s_{!}\left(q_{1}^{-1} F \stackrel{\mathrm{L}}{\otimes} q_{2}^{-1} G\right) \simeq \mathrm{R} s_{!} \delta_{M}^{-1}(F \otimes \stackrel{\mathrm{L}}{\otimes} G), \\
F \star_{n p} G & :=\mathrm{R} s_{*}\left(q_{1}^{-1} F \stackrel{\mathrm{L}}{\otimes} q_{2}^{-1} G\right) \simeq \mathrm{R} s_{*} \delta_{M}^{-1}(F \otimes G) .
\end{aligned}
$$

The morphism $\mathbf{k}_{\gamma} \rightarrow \mathbf{k}_{M \times\{0\}}$ gives the morphism

$$
F \star_{n p} \mathbf{k}_{\gamma} \rightarrow F
$$

Recall the following result:

Proposition 3.9. (Microlocal cut-off lemma [5, Prop. 5.2.3, 3.5.4]) Let $F \in$ $\mathrm{D}^{\mathrm{b}}\left(\mathbf{k}_{E}\right)$. Then $\mathrm{SS}(F) \subset T^{*} M \times V \times \gamma_{0}^{\circ}$ if and only if the morphism (43) is an isomorphism.

If $\gamma_{0}$ has a non-empty interior we have $\mathbf{k}_{\gamma_{0}} \simeq \mathrm{D}_{V}^{\prime}\left(\mathbf{k}_{\operatorname{Int} \gamma_{0}}\right)$ and we deduce from Corollary 1.7 (ii) that

$$
F \star_{n p} \mathbf{k}_{\gamma} \simeq \mathrm{R} s_{*} \mathrm{R} \Gamma_{M \times V \times \operatorname{Int} \gamma_{0}}\left(q_{1}^{-1} F\right) .
$$

Following Tamarkin [12, we introduce a right adjoint to the convolution functor by setting for $F, G \in \mathrm{D}^{\mathrm{b}}\left(\mathbf{k}_{E}\right)$

$$
\mathscr{H o m}^{*}(G, F):=\operatorname{R} q_{1 *} \mathrm{R} \mathscr{H} \text { om }\left(q_{2}^{-1} G, s^{!} F\right) .
$$

Hence for $F_{1}, F_{2}, F_{3} \in \mathrm{D}^{\mathrm{b}}\left(\mathbf{k}_{E}\right)$, we have

$$
\operatorname{RHom}\left(F_{1} \star F_{2}, F_{3}\right) \simeq \operatorname{RHom}\left(F_{1}, \mathscr{H o m}^{*}\left(F_{2}, F_{3}\right)\right) \text {. }
$$

We use the notation:

$$
i: E \rightarrow E \text { denotes the involution }(x, y) \mapsto(x,-y) .
$$


Lemma 3.10. For $F, G \in \mathrm{D}^{\mathrm{b}}\left(\mathbf{k}_{E}\right)$ we have

$$
\begin{aligned}
\mathscr{H} o m^{*}(G, F) & \simeq \operatorname{R} s_{*} \operatorname{R} \mathscr{H} o m\left(q_{2}^{-1} i^{-1} G, q_{1}^{!} F\right), \\
F \star G & \simeq \operatorname{R} q_{1 !}\left(s^{-1} F \stackrel{\mathrm{L}}{\otimes} q_{2}^{-1} i^{-1} G\right) .
\end{aligned}
$$

Proof. We only prove the first isomorphism, the second one being similar. We set $f:=\left(s,-q_{2}\right): E \times_{M} E \rightarrow E \times_{M} E,\left(x, v_{1}, v_{2}\right) \mapsto\left(x, v_{1}+v_{2},-v_{2}\right)$. We find $f \circ f=\mathrm{id}, s=q_{1} \circ f, q_{2} \circ f=i \circ q_{2}$. Since $f$ is an isomorphism R $\mathscr{H} \circ \mathrm{om}$ commutes with $f^{-1} \simeq f^{!}$. Since $f \circ f=$ id we have $f^{-1}=f_{*}$. We deduce the isomorphisms:

$$
\begin{aligned}
\mathscr{H o m}^{*}(G, F) & \simeq \operatorname{R} q_{1 *} \operatorname{R} \mathscr{H} o m\left(q_{2}^{-1} G, s^{!} F\right) \\
& \simeq \operatorname{R} q_{1 *} \operatorname{R} \mathscr{H} o m\left(f^{-1} q_{2}^{-1} i^{-1} G, f^{!} q_{1}^{!} F\right) \\
& \simeq \operatorname{R} q_{1 *} f^{-1} \operatorname{R} \mathscr{H} o m\left(q_{2}^{-1} i^{-1} G, q_{1}^{!} F\right) \\
& \simeq \operatorname{R} s_{*} \operatorname{R} \mathscr{H} o m\left(q_{2}^{-1} i^{-1} G, q_{1}^{!} F\right) .
\end{aligned}
$$

Proposition 3.11. For $F_{1}, F_{2}, F_{3} \in \mathrm{D}^{\mathrm{b}}\left(\mathbf{k}_{E}\right)$ we have

$$
\begin{aligned}
\left(F_{1} \star F_{2}\right) \star F_{3} & \simeq F_{1} \star\left(F_{2} \star F_{3}\right), \\
\mathscr{H o m}^{*}\left(F_{1} \star F_{2}, F_{3}\right) & \simeq \mathscr{H o m}^{*}\left(F_{1}, \mathscr{H}^{*} m^{*}\left(F_{2}, F_{3}\right)\right) .
\end{aligned}
$$

Proof. (i) The first isomorphism is proved in the same way as the associativity of the composition of kernels: we check easily that both sides are isomorphic to $\mathrm{R} \sigma_{!}\left(q_{1}^{-1}\left(F_{1}\right) \stackrel{\mathrm{L}}{\otimes} q_{2}^{-1}\left(F_{2}\right) \stackrel{\mathrm{L}}{\otimes} q_{3}^{-1}\left(F_{3}\right)\right)$ where $\sigma: M \times V^{3} \rightarrow M \times V$ is given by $\sigma\left(x, v_{1}, v_{2}, v_{3}\right)=\left(x, v_{1}+v_{2}+v_{3}\right)$ and $q_{i}: M \times V^{3} \rightarrow M \times V$ is the projection on the $i^{\text {th }}$ factor $V$.

(ii) We use the Yoneda embedding to prove the second isomorphism. We apply the functor $\operatorname{Hom}_{\mathrm{D}^{\mathrm{b}}\left(\mathbf{k}_{E}\right)}(H, \bullet)$ for any $H \in \mathrm{D}^{\mathrm{b}}\left(\mathbf{k}_{E}\right)$ to each term of this formula. One gets an isomorphism in view of the adjunction isomorphism (46) and the associativity of $\star$ proved in (i).

Proposition 3.12. Let $q: E \rightarrow M$ and $q^{\prime}: M \times V \times V \rightarrow M$ be the projections. For $F, G, H \in \mathrm{D}^{\mathrm{b}}\left(\mathbf{k}_{E}\right)$ we have

$$
\begin{aligned}
& \mathrm{R} q_{*}\left(\operatorname{R} \mathscr{H} o m\left(F, \mathscr{H} o m^{*}(G, H)\right)\right) \simeq \operatorname{R} q_{*}(\mathrm{R} \mathscr{H} o m(F \star G, H)), \\
& \mathrm{R} q_{!}((F \star G) \stackrel{\mathrm{L}}{\otimes} H) \simeq \mathrm{R} q_{!}^{\prime}\left(q_{1}^{-1} F \stackrel{\mathrm{L}}{\otimes} q_{2}^{-1} G \stackrel{\mathrm{L}}{\otimes} s^{-1} H\right) \\
& \simeq \mathrm{R} q_{!}\left(F \stackrel{\mathrm{L}}{\otimes}\left(i^{-1} G \star H\right)\right) \text {. }
\end{aligned}
$$


Proof. The first isomorphism follows by adjunction from (41) and (45), using $q \circ q_{1}=q \circ s$. The second and third ones follow from the projection formula, the identities $q \circ q_{1}=q^{\prime}=q \circ s$ and Lemma 3.10.

Recall that the involution $(\cdot)^{\alpha}$ is defined in (37).

Proposition 3.13. For $F, G \in \mathrm{D}^{\mathrm{b}}\left(\mathbf{k}_{E}\right)$ we have

$$
\begin{aligned}
& \mathrm{SS}(F \star G) \subset \mathrm{SS}(F) \widehat{\mathrm{SS}}(G), \\
& \mathrm{SS}\left(\mathscr{H} \text { om }^{*}(G, F)\right) \subset \mathrm{SS}(F) \widehat{ } \mathrm{SS}(G)^{\alpha} .
\end{aligned}
$$

Proof. Both inclusions in (51) follow from (41), (38) and Theorems 1.12 and 1.16. For the second one we also use Lemma 3.10 and $\operatorname{SS}\left(i^{-1} G\right)^{a}=$ $\mathrm{SS}(G)^{\alpha}$.

Using (51) and (39), we get:

Corollary 3.14. Let $F, G \in \mathrm{D}^{\mathrm{b}}\left(\mathbf{k}_{E}\right)$ and assume that there exist closed cones $A^{\prime}, B^{\prime} \subset V^{*}$ such that $\mathrm{SS}(F) \subset T^{*} M \times V \times A^{\prime}$ and $\mathrm{SS}(G) \subset T^{*} M \times V \times B^{\prime}$. Then

$$
\begin{aligned}
& \mathrm{SS}(F \star G) \subset T^{*} M \times V \times\left(A^{\prime} \cap B^{\prime}\right), \\
& \operatorname{SS}\left(\mathscr{H}_{o m^{*}}(G, F)\right) \subset T^{*} M \times V \times\left(A^{\prime} \cap B^{\prime}\right) .
\end{aligned}
$$

Corollary 3.15. Let $F, G \in \mathrm{D}^{\mathrm{b}}\left(\mathbf{k}_{E}\right)$ and assume that there exist closed strict $\gamma$-cones $A$ and $B$ in $T^{*} M \times V^{*}$ such that $\operatorname{SS}(F) \subset \widehat{\pi}_{E}^{-1}(A)$ and $\operatorname{SS}(G) \subset$ $\widehat{\pi}_{E}^{-1}(B)$. Let $N$ be a submanifold of $M$ and $j: N \times V \rightarrow M \times V$ the inclusion. Then

$$
j^{-1} \mathscr{H}_{o m}^{*}(F, G) \simeq \mathscr{H}_{o m}^{*}\left(j^{-1} F, j^{-1} G\right) .
$$

Proof. By Proposition 3.13 and Lemma 3.7. $\operatorname{SS}\left(\mathscr{H}_{0 m}^{*}(F, G)\right) \subset \widehat{\pi}_{E}^{-1}(A+B)$ and $A+B$ is a strict $\gamma$-cone. By Remark 3.3, we deduce $j^{!} H \simeq j^{-1} H \otimes$ $\omega_{N \times V \mid M \times V}$ for $H=F, G$ or $\mathscr{H}_{o m^{*}}(F, G)$. This gives the first and last steps in the sequence of isomorphisms, where we set $j^{\prime}=j \times \mathrm{id}_{V}$ :

$$
\begin{aligned}
j^{-1} \mathscr{H o m}^{*}(F, G) & \simeq j^{!} \mathrm{R} q_{1 *} \operatorname{R} \mathscr{H} o m\left(q_{2}^{-1} F, s^{!} G\right) \otimes \omega_{N \times V \mid M \times V}^{\otimes-1} \\
& \simeq \operatorname{R} q_{1_{*}} j^{\prime !} \operatorname{RH} o m\left(q_{2}^{-1} F, s^{!} G\right) \otimes \omega_{N \times V \mid M \times V}^{\otimes-1} \\
& \simeq \operatorname{R} q_{1_{*}} \operatorname{R} \mathscr{H} o m\left(j^{\prime-1} q_{2}^{-1} F, j^{\prime !} s^{!} G\right) \otimes \omega_{N \times V \mid M \times V}^{\otimes-1} \\
& \simeq \operatorname{R} q_{1 *} \operatorname{R} \mathscr{H} o m\left(q_{2}^{-1} j^{-1} F, s^{!} j^{!} G\right) \otimes \omega_{N \times V \mid M \times V}^{\otimes-1} \\
& \simeq \mathscr{H} o m^{*}\left(j^{-1} F, j^{-1} G\right) .
\end{aligned}
$$




\section{Kernels associated with cones}

Recall that we consider a trivial vector bundle $E=M \times V$ and a trivial cone $\gamma=M \times \gamma_{0}$ satisfying (34). For another proper closed convex cone $\lambda_{0} \subset V$ such that $\lambda_{0} \subset \gamma_{0}$, setting $\lambda=M \times \lambda_{0}$, we shall use the exact sequence of sheaves:

$$
0 \rightarrow \mathbf{k}_{\gamma \backslash \lambda} \rightarrow \mathbf{k}_{\gamma} \rightarrow \mathbf{k}_{\lambda} \rightarrow 0
$$

Lemma 3.16. Let $\lambda_{0} \subset \gamma_{0}$ be closed convex proper cones. Then

$$
\begin{aligned}
& \mathrm{SS}\left(\mathbf{k}_{\gamma}\right) \subset T_{M}^{*} M \times V \times \gamma_{0}^{\circ} \\
& \mathrm{SS}\left(\mathbf{k}_{\gamma \backslash \lambda}\right) \subset T_{M}^{*} M \times V \times\left(\lambda_{0}^{\circ} \backslash \operatorname{Int}\left(\gamma_{0}^{\circ}\right)\right) .
\end{aligned}
$$

Proof. Since our sheaves are inverse images of sheaves on $V$ we may as well assume that $M$ is a point. Since our sheaves are conic in the sense of [5, §5.5] their microsupports are biconic. Now, a closed biconic subset $A$ of $V \times V^{*}$ satisfies $A \subset V \times\left(A \cap\{0\} \times V^{*}\right)$. Hence we only have to check the inclusions at the origin.

Then the first inclusion follows from [5, Prop. 5.3.1].

For the second one we use the Sato-Fourier transform $(\cdot)^{\wedge}: \mathrm{D}_{\mathbb{R}^{+}}^{\mathrm{b}}\left(\mathbf{k}_{V}\right) \rightarrow$ $\mathrm{D}_{\mathbb{R}^{+}}^{\mathrm{b}}\left(\mathbf{k}_{V^{*}}\right)$ defined in [5, §3.7] $\left(\mathrm{D}_{\mathbb{R}^{+}}^{\mathrm{b}}\left(\mathbf{k}_{V}\right)\right.$ denotes the subcategory of complexes with conic cohomology). We have $\left(\mathbf{k}_{\gamma_{0}}\right)^{\wedge} \simeq \mathbf{k}_{\operatorname{Int} \gamma_{0}^{\circ}}$ and we deduce the distinguished triangle

$$
\left(\mathbf{k}_{\gamma_{0} \backslash \lambda_{0}}\right)^{\wedge} \rightarrow \mathbf{k}_{\operatorname{Int} \gamma_{0}^{\circ}} \rightarrow \mathbf{k}_{\operatorname{Int} \lambda_{0}^{\circ}} \stackrel{+1}{\longrightarrow} .
$$

Hence $\left(\mathbf{k}_{\gamma_{0} \backslash \lambda_{0}}\right)^{\wedge} \simeq \mathbf{k}_{\operatorname{Int} \lambda_{0}^{\circ} \backslash \operatorname{Int} \gamma_{0}^{\circ}}[-1]$ and we conclude with [5, Prop. 5.5.5] which implies $\operatorname{SS}(F) \cap T_{0}^{*} V=\operatorname{supp}\left(F^{\wedge}\right)$ for $F \in \mathrm{D}_{\mathbb{R}^{+}}^{\mathrm{b}}\left(\mathbf{k}_{V}\right)$.

We introduce the kernel:

$$
L_{\gamma}:=\mathbf{k}_{\gamma} \star: \mathrm{D}^{\mathrm{b}}\left(\mathbf{k}_{E}\right) \rightarrow \mathrm{D}^{\mathrm{b}}\left(\mathbf{k}_{E}\right) .
$$

The morphism $\mathbf{k}_{\gamma} \rightarrow \mathbf{k}_{\{0\}}$ induces a morphism of functors $\varepsilon: L_{\gamma} \rightarrow \operatorname{id}_{\mathrm{D}^{\mathrm{b}}\left(\mathbf{k}_{E}\right)}$. By (48) we have $L_{\gamma} \circ L_{\gamma} \simeq L_{\gamma}$. Hence, the pair $\left(L_{\gamma}, \varepsilon\right)$ is a projector in $\mathrm{D}^{\mathrm{b}}\left(\mathbf{k}_{E}\right)^{\text {op }}$ in the sense of [6, Chap. 5]. It will be convenient to write $L_{\gamma}$ with the language of kernels as in (77). We define $\gamma^{+} \subset E \times E$ by

$$
\gamma^{+}=\left\{\left(x, v, x^{\prime}, v^{\prime}\right) \in E \times E ; v-v^{\prime} \in \gamma_{0}\right\}
$$


Then

$$
L_{\gamma} \simeq \mathbf{k}_{\gamma^{+}} \circ \cdot
$$

In the sequel we set

$$
\begin{aligned}
& U_{\gamma}:=T^{*} M \times V \times \operatorname{Int}\left(\gamma_{0}^{\circ}\right), \\
& Z_{\gamma}:=T^{*} E \backslash U_{\gamma} .
\end{aligned}
$$

Proposition 3.17. Let $F \in \mathrm{D}^{\mathrm{b}}\left(\mathbf{k}_{E}\right)$.

(i) $\mathrm{SS}\left(L_{\gamma} F\right) \subset \overline{U_{\gamma}}=T^{*} M \times V \times \gamma_{0}^{\circ}$.

(ii) Consider a distinguished triangle $L_{\gamma} F \rightarrow F \rightarrow G \stackrel{+1}{\longrightarrow}$. Then $\operatorname{SS}(G) \subset$ $Z_{\gamma}$. In particular, $\mathrm{SS}\left(L_{\gamma} F\right) \subset\left(T^{*} M \times V \times \partial \gamma_{0}^{\circ}\right) \cup\left(\mathrm{SS}(F) \cap U_{\gamma}\right)$.

(iii) Let $G \in \mathrm{D}_{Z_{\gamma}}^{\mathrm{b}}\left(\mathbf{k}_{E}\right)$. Then $\mathrm{R}_{q_{*}} \mathrm{R} \Gamma_{\gamma}(G) \simeq 0$. In particular, $\mathrm{R} \Gamma_{\gamma}(E ; G) \simeq$ 0 .

Proof. (i) follows from (52) and Lemma 3.16.

(ii) Using the exact sequence (53), we have $G \simeq \mathbf{k}_{\gamma \backslash\{0\}} \star F$. Then the result again follows from (52) and Lemma 3.16.

(iii) We set $H=\mathrm{R} \Gamma_{\gamma}(G) \simeq \operatorname{R} \mathscr{H}$ om $\left(\mathbf{k}_{\gamma}, G\right)$. It follows from Theorem 1.12 that $\operatorname{SS}(H) \subset Z_{\gamma}$. Choose a vector $\xi \in \operatorname{Int}\left(\gamma_{0}^{\circ}\right)$ and consider the projection

$$
\theta: M \times V \rightarrow M \times \mathbb{R}, \quad \theta(x, v)=(x ;\langle\xi, v\rangle) .
$$

Since $\gamma$ is a proper cone, $\theta$ is proper on supp $H$ and we get by Theorem 1.6 that $\operatorname{SS}\left(\operatorname{R} \theta_{*}(H)\right) \subset\{\tau \leq 0\}$ where $(t ; \tau)$ are the coordinates on $T^{*} \mathbb{R}$. Moreover, $\operatorname{supp} \operatorname{R} \theta_{*}(H) \subset M \times\{t \geq 0\}$.

Now it is enough to prove that $\mathrm{R} \Gamma\left(U \times \mathbb{R} ; \mathrm{R} \theta_{*}(H)\right)=0$, for any open subset $U$ of $M$. Denote by $p: U \times \mathbb{R} \rightarrow \mathbb{R}$ the projection and set $\widetilde{H}=$ $\mathrm{R} p_{*} \mathrm{R} \theta_{*}(H)$. Although $p$ is not proper on $\operatorname{supp}(\widetilde{H})$, one easily checks that $\operatorname{SS}(\widetilde{H}) \subset\{t \geq 0, \tau \leq 0\}$ and this implies $\widetilde{H} \simeq 0$. (This is a special case of Corollary 1.8.)

The next Lemma follows immediately from the adjunction formula (46).

Lemma 3.18. Let $F, G \in \mathrm{D}^{\mathrm{b}}\left(\mathbf{k}_{E}\right)$ and assume that $L_{\gamma} F \stackrel{\sim}{\longrightarrow}$. Then we have $\operatorname{Hom}_{\mathrm{D}^{\mathrm{b}}\left(\mathbf{k}_{E}\right)}(F, G) \simeq \mathrm{R} \Gamma_{\gamma}\left(E ; \mathscr{H}_{o m}{ }^{*}(F, G)\right)$. 
Proposition 3.19. (a) Let $F \in \mathrm{D}^{\mathrm{b}}\left(\mathbf{k}_{E}\right)$. Then $F \in \mathrm{D}_{Z_{\gamma}}^{\mathrm{b}}\left(\mathbf{k}_{E}\right)^{\perp, l}$ if and only if the natural morphism $L_{\gamma} F \rightarrow F$ is an isomorphism.

(b) Let $G \in \mathrm{D}_{Z_{\gamma}}^{\mathrm{b}}\left(\mathbf{k}_{E}\right)$. Then $L_{\gamma} G \simeq 0$.

Proof. (a)-(i) Assume $F \simeq L_{\gamma} F$. Let $G \in \mathrm{D}_{Z_{\gamma}}^{\mathrm{b}}\left(\mathbf{k}_{E}\right)$ and set $H:=\mathscr{H}_{0}{ }^{*}(F, G)$. Then $H$ belongs to $\mathrm{D}_{Z_{\gamma}}^{\mathrm{b}}\left(\mathbf{k}_{E}\right)$ by (52) and $\mathrm{R} \Gamma_{\gamma}(E ; H) \simeq 0$ by Proposition 3.17. Since $F \simeq L_{\gamma} F$, we get $\operatorname{Hom}_{\mathrm{D}^{\mathrm{b}}\left(\mathbf{k}_{E}\right)}(F, G)=0$ by Lemma 3.18 .

(a)-(ii) Assume that $F \in \mathrm{D}_{Z_{\gamma}}^{\mathrm{b}}\left(\mathbf{k}_{E}\right)^{\perp, l}$ and consider a distinguished triangle $L_{\gamma} F \rightarrow F \rightarrow G \stackrel{+1}{\rightarrow}$. By (a)-(i) $L_{\gamma} F$ also belongs to $D_{Z_{\gamma}}^{\mathrm{b}}\left(\mathbf{k}_{E}\right)^{\perp, l}$. Hence so does $G$. On the other hand, $G \in \mathrm{D}_{Z_{\gamma}}^{\mathrm{b}}\left(\mathbf{k}_{E}\right)$ by Proposition 3.17. Hence, $G \simeq 0$.

(b) Let $G \in \mathrm{D}_{Z_{\gamma}}^{\mathrm{b}}\left(\mathbf{k}_{E}\right)$ and consider a distinguished triangle $L_{\gamma} G \rightarrow G \rightarrow$ $H \stackrel{+1}{\longrightarrow}$. Since both $G$ and $H$ belong to $\mathrm{D}_{Z_{\gamma}}^{\mathrm{b}}\left(\mathbf{k}_{E}\right)$, so does $L_{\gamma} G$. Since $L_{\gamma} G$ belongs to $\mathrm{D}_{Z_{\gamma}}^{\mathrm{b}}\left(\mathbf{k}_{E}\right)^{\perp, l}$, it is 0 .

Remark 3.20. One can also consider the projector

$$
R_{\gamma}:=\mathscr{H o m}^{*}\left(\mathbf{k}_{\gamma}, \cdot\right): \mathrm{D}^{\mathrm{b}}\left(\mathbf{k}_{E}\right) \rightarrow \mathrm{D}^{\mathrm{b}}\left(\mathbf{k}_{E}\right) .
$$

Then we obtain similar results to Propositions 3.17, 3.19 and Lemma 3.18 with $R_{\gamma}$ instead of $L_{\gamma}$. Note that the pair $\left(L_{\gamma}, R_{\gamma}\right)$ is a pair of adjoint functors:

$$
\begin{aligned}
\operatorname{Hom}_{\mathrm{D}^{\mathrm{b}}\left(\mathbf{k}_{E}\right)}\left(L_{\gamma} F, G\right) & \simeq \operatorname{Hom}_{\mathrm{D}^{\mathrm{b}}\left(\mathbf{k}_{E}\right)}\left(F, R_{\gamma} G\right) \\
& \simeq \operatorname{Hom}_{\mathrm{D}^{\mathrm{b}}\left(\mathbf{k}_{E}\right)}\left(\mathbf{k}_{\gamma}, \mathscr{H}_{o m^{*}}(F, G)\right) .
\end{aligned}
$$

Note that $\mathbf{k}_{\gamma}$ is cohomologically constructible. If we assume that $\operatorname{Int}(\gamma) \neq \emptyset$, then $\mathrm{D}^{\prime} \mathbf{k}_{\gamma} \simeq \mathbf{k}_{\operatorname{Int}(\gamma)}$ and one deduces from Lemma 3.10 that

$$
\mathscr{H} o m^{*}\left(\mathbf{k}_{\gamma}, \mathbf{k}_{\gamma}\right) \simeq \mathbf{k}_{\operatorname{Int}(-\gamma)}\left[d_{V}\right]
$$

where $d_{V}$ is the dimension of $V$.

\section{Projector and localization}

Recall that $E=M \times V$ is a trivial vector bundle over $M, \gamma_{0}$ is a cone satisfying (34) and the sets $U_{\gamma}$ and $Z_{\gamma}$ are defined in (57). By definition $\mathrm{D}^{\mathrm{b}}\left(\mathbf{k}_{E} ; U_{\gamma}\right)$ is a localization of $\mathrm{D}^{\mathrm{b}}\left(\mathbf{k}_{E}\right)$ and we let $Q_{\gamma}: \mathrm{D}^{\mathrm{b}}\left(\mathbf{k}_{E}\right) \rightarrow \mathrm{D}^{\mathrm{b}}\left(\mathbf{k}_{E} ; U_{\gamma}\right)$ be the functor of localization. 
Proposition 3.21. (i) The functor $L_{\gamma}$ defined in (54) takes its values in $\mathrm{D}_{Z_{\gamma}}^{\mathrm{b}}\left(\mathbf{k}_{E}\right)^{\perp, l}$ and sends $\mathrm{D}_{Z_{\gamma}}^{\mathrm{b}}\left(\mathbf{k}_{E}\right)$ to 0 . It factorizes through $Q_{\gamma}$ and induces a functor $l_{\gamma}: \mathrm{D}^{\mathrm{b}}\left(\mathbf{k}_{E} ; U_{\gamma}\right) \rightarrow \mathrm{D}^{\mathrm{b}}\left(\mathbf{k}_{E}\right)$ such that $L_{\gamma} \simeq l_{\gamma} \circ Q_{\gamma}$.

(ii) The functor $l_{\gamma}$ is left adjoint to $Q_{\gamma}$ and induces an equivalence $\mathrm{D}^{\mathrm{b}}\left(\mathbf{k}_{E} ; U_{\gamma}\right) \simeq \mathrm{D}_{Z_{\gamma}}^{\mathrm{b}}\left(\mathbf{k}_{E}\right)^{\perp, l}$.

This is visualized by the diagram

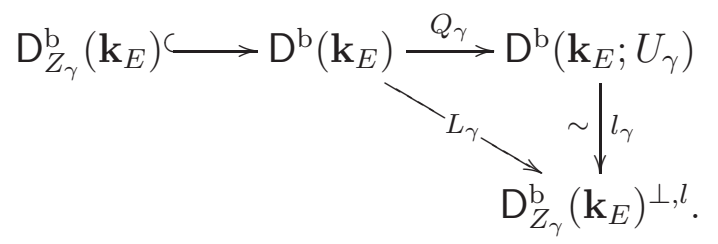

Proof. This follows from Proposition 3.19 together with the classical results on the localization of triangulated categories recalled in Section 1 (see e.g., [6, Exe. 10.15]).

In particular, we have for $F, G \in \mathrm{D}^{\mathrm{b}}\left(\mathbf{k}_{E}\right)$

$$
\begin{aligned}
\operatorname{Hom}_{\mathrm{D}^{\mathrm{b}}\left(\mathbf{k}_{E} ; U_{\gamma}\right)}\left(Q_{\gamma}(F), Q_{\gamma}(G)\right) & \simeq \operatorname{Hom}_{\mathrm{D}^{\mathrm{b}}\left(\mathbf{k}_{E}\right)}\left(L_{\gamma}(F), G\right) \\
& \simeq \operatorname{Hom}_{\mathrm{D}^{\mathrm{b}}\left(\mathbf{k}_{E}\right)}\left(L_{\gamma}(F), L_{\gamma}(G)\right) .
\end{aligned}
$$

There is a similar result to Proposition 3.21, replacing the functor $L_{\gamma}$ with the functor $R_{\gamma}$. The functor $R_{\gamma}$ takes its values in $\mathrm{D}_{Z_{\gamma}}^{\mathrm{b}}\left(\mathbf{k}_{E}\right)^{\perp, r}$ and sends $\mathrm{D}_{Z_{\gamma}}^{\mathrm{b}}\left(\mathbf{k}_{E}\right)$ to 0 . It factorizes through $Q_{\gamma}$ and induces a functor $r_{\gamma}: \mathrm{D}^{\mathrm{b}}\left(\mathbf{k}_{E} ; U_{\gamma}\right) \rightarrow$ $\mathrm{D}^{\mathrm{b}}\left(\mathbf{k}_{E}\right)$ such that $R_{\gamma} \simeq r_{\gamma} \circ Q_{\gamma}$.

We notice that, for $F \in \mathrm{D}_{Z_{\gamma}}^{\mathrm{b}}\left(\mathbf{k}_{E}\right)^{\perp, l}$ or $G \in \mathrm{D}_{Z_{\gamma}}^{\mathrm{b}}\left(\mathbf{k}_{E}\right)^{\perp, r}$, we have

$$
\mathscr{H}_{o m}^{*}(F, G) \in \mathrm{D}_{Z_{\gamma}}^{\mathrm{b}}\left(\mathbf{k}_{E}\right)^{\perp, r}
$$

By Proposition 3.17 (used with $R_{\gamma}$ instead of $L_{\gamma}$ ) we obtain in particular

$$
\mathscr{H}_{o m}^{*}(F, G) \in \mathrm{D}_{\overline{U_{\gamma}}}^{\mathrm{b}}\left(\mathbf{k}_{M}\right) \text {. }
$$

Notation 3.22. Let us set for short

$$
\begin{aligned}
\mathrm{D}^{\mathrm{b}}\left(\mathbf{k}_{M}^{\gamma}\right) & :=\mathrm{D}^{\mathrm{b}}\left(\mathbf{k}_{E} ; U_{\gamma}\right), \\
\mathrm{D}^{\mathrm{b}}\left(\mathbf{k}_{M}^{\gamma, l}\right) & :=\mathrm{D}_{Z_{\gamma}}^{\mathrm{b}}\left(\mathbf{k}_{E}\right)^{\perp, l}, \\
\mathrm{D}^{\mathrm{b}}\left(\mathbf{k}_{M}^{\gamma, r}\right) & :=\mathrm{D}_{Z_{\gamma}}^{\mathrm{b}}\left(\mathbf{k}_{E}\right)^{\perp, r} .
\end{aligned}
$$


When $M=$ pt, we set

$$
\mathrm{D}^{\mathrm{b}}\left(\mathbf{k}^{\gamma}\right):=\mathrm{D}^{\mathrm{b}}\left(\mathbf{k}_{\mathrm{pt}}^{\gamma}\right)
$$

and similarly with $\mathrm{D}^{\mathrm{b}}\left(\mathbf{k}^{\gamma, l}\right)$ and $\mathrm{D}^{\mathrm{b}}\left(\mathbf{k}^{\gamma, r}\right)$.

Denote by $p: E=M \times V \rightarrow V$ the projection and denote by $\Gamma^{\gamma}$ the functor

$$
\Gamma^{\gamma}(\bullet)=\operatorname{RHom}\left(\mathbf{k}_{\gamma}, \bullet\right): \mathrm{D}^{\mathrm{b}}\left(\mathbf{k}^{\gamma}\right) \rightarrow \mathrm{D}^{\mathrm{b}}(\mathbf{k}) .
$$

We get the diagram of categories in which the horizontal arrows are equivalences

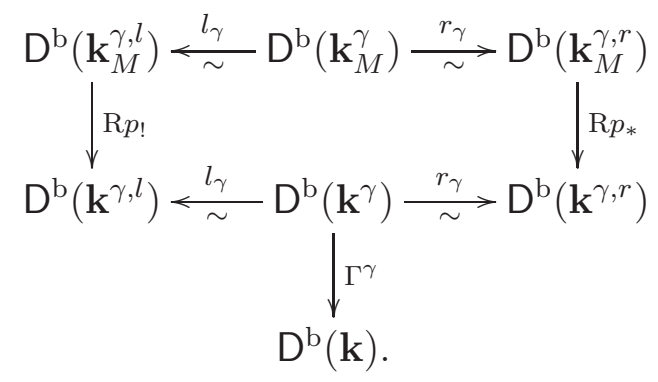

Note that by Lemma 3.18 , for $F \in \mathrm{D}^{\mathrm{b}}\left(\mathbf{k}_{M}^{\gamma, l}\right)$ or $G \in \mathrm{D}^{\mathrm{b}}\left(\mathbf{k}_{M}^{\gamma, r}\right)$, we have

$$
\operatorname{RHom}_{\mathrm{D}^{\mathrm{b}}\left(\mathbf{k}_{M}^{\gamma}\right)}(F, G) \simeq \Gamma^{\gamma} \circ \operatorname{Rp}_{*} \mathscr{H}_{o m^{*}}(F, G) .
$$

Embedding the category $\mathrm{D}^{\mathrm{b}}\left(\mathbf{k}_{M}\right)$ into $\mathrm{D}^{\mathrm{b}}\left(\mathbf{k}_{M}^{\gamma}\right)$

Recall that $q: E \rightarrow M$ denotes the projection and consider the functor

$$
\Psi_{\gamma}: \mathrm{D}^{\mathrm{b}}\left(\mathbf{k}_{M}\right) \rightarrow \mathrm{D}^{\mathrm{b}}\left(\mathbf{k}_{E}\right), \quad F \mapsto q^{-1} F \otimes \mathbf{k}_{\gamma} .
$$

Lemma 3.23. One has the isomorphism of functors $L_{\gamma} \circ \Psi_{\gamma} \sim \Psi_{\gamma}$.

Proof. One has

$$
\begin{aligned}
L_{\gamma} \circ \Psi_{\gamma}(F) & =\operatorname{R} s_{!}\left(q_{1}^{-1} \mathbf{k}_{\gamma} \otimes q_{2}^{-1}\left(q^{-1} F \otimes \mathbf{k}_{\gamma}\right)\right) \\
& \simeq \operatorname{R} s_{!}\left(q_{1}^{-1} \mathbf{k}_{\gamma} \otimes q_{2}^{-1} \mathbf{k}_{\gamma} \otimes q_{2}^{-1}\left(q^{-1} F\right)\right) \\
& \simeq \operatorname{R} s_{!}\left(q_{1}^{-1} \mathbf{k}_{\gamma} \otimes q_{2}^{-1} \mathbf{k}_{\gamma} \otimes s^{-1}\left(q^{-1} F\right)\right) \\
& \simeq \operatorname{R} s_{!}\left(q_{1}^{-1} \mathbf{k}_{\gamma} \otimes q_{2}^{-1} \mathbf{k}_{\gamma}\right) \otimes q^{-1} F \\
& \simeq \mathbf{k}_{\gamma} \otimes q^{-1} F .
\end{aligned}
$$


In the sequel, we consider $\Psi_{\gamma}$ as a functor

$$
\Psi_{\gamma}: \mathrm{D}^{\mathrm{b}}\left(\mathbf{k}_{M}\right) \rightarrow \mathrm{D}^{\mathrm{b}}\left(\mathbf{k}_{M}^{\gamma}\right) .
$$

Proposition 3.24. The functor $\Psi_{\gamma}$ in (69) is fully faithful.

Proof. Let $F, G \in \mathrm{D}^{\mathrm{b}}\left(\mathbf{k}_{M}\right)$. Then

$$
\begin{aligned}
\operatorname{Hom}_{\mathrm{D}^{\mathrm{b}}\left(\mathbf{k}_{E}\right)}\left(\mathbf{k}_{\gamma} \otimes q^{-1} G, \mathbf{k}_{\gamma} \otimes\right. & \left.q^{-1} F\right) \\
& \simeq \operatorname{Hom}_{\mathrm{D}^{\mathrm{b}\left(\mathbf{k}_{M}\right)}}\left(G, \operatorname{R} q_{*} \operatorname{R} \mathscr{H} o m\left(\mathbf{k}_{\gamma}, q^{-1} F \otimes \mathbf{k}_{\gamma}\right)\right) \\
& \simeq \operatorname{Hom}_{\mathrm{D}^{\mathrm{b}\left(\mathbf{k}_{M}\right)}}\left(G, \operatorname{R} q_{*}\left(q^{-1} F \otimes \mathbf{k}_{\gamma}\right)\right) .
\end{aligned}
$$

Hence, it is enough to check the isomorphism

$$
F \stackrel{\sim}{\longrightarrow} q_{*}\left(q^{-1} F \otimes \mathbf{k}_{\gamma}\right) .
$$

Denote by $\widetilde{q}$ the projection $\gamma \rightarrow M$. The isomorphism (70) reduces to

$$
F \simeq \mathrm{R} \widetilde{q}_{*} \widetilde{q}^{-1} F
$$

and this last isomorphism follows from the fact that $\gamma$ is a closed convex cone, hence is contractible (see for example [5, Prop. 2.7.8]).

\section{A cut-off result}

Recall that we consider a trivial vector bundle $E=M \times V$ and a trivial cone $\gamma=M \times \gamma_{0}$ satisfying (34). We also recall that a subset of $T^{*} M \times V^{*}$ is a cone if it is stable by the action (17). The map $\widehat{\pi}$ is defined in (16) and we have set (see (57)):

$$
U_{\gamma}=T^{*} M \times V \times \operatorname{Int} \gamma_{0}^{\circ} .
$$

By the equivalence $l_{\gamma}$ of Proposition [3.21, any object $F \in \mathrm{D}^{\mathrm{b}}\left(\mathbf{k}_{E} ; U_{\gamma}\right)$ has a canonical representative in $\mathrm{D}^{\mathrm{b}}\left(\mathbf{k}_{E}\right)$ again denoted by $F$ and we have $F \simeq$ $L_{\gamma}(F)$. By Proposition 3.17 (i) we have $\mathrm{SS}(F) \subset \overline{U_{\gamma}}$.

We first state a kind of cut-off lemma in the case where $M$ is a point.

Lemma 3.25. Let $V$ be a vector space and $\gamma \subset V$ a closed convex proper cone containing 0 . Set $U_{\gamma}:=V \times \operatorname{Int} \gamma^{\circ}$ and $Z_{\gamma}:=T^{*} V \backslash U_{\gamma}$. Let $F \in \mathrm{D}_{Z_{\gamma}}^{\mathrm{b}}\left(\mathbf{k}_{V}\right)^{\perp, l}$. We assume that there exists a closed cone $A \subset V^{*}$ such that 
(i) $A \subset \operatorname{Int} \gamma^{\circ} \cup\{0\}$,

(ii) $\mathrm{SS}(F) \cap U_{\gamma} \subset V \times A$.

Then $\operatorname{SS}(F) \subset\left(\mathrm{SS}(F) \cap U_{\gamma}\right) \cup T_{V}^{*} V$.

Proof. (i) Up to enlarging $A$ we may as well assume that $\operatorname{SS}(F) \cap U_{\gamma} \subset$ $V \times \operatorname{Int} A$. We set $\lambda=A^{\circ}$. Hence $\lambda$ is a closed convex proper cone of $V$ and we have

$$
\begin{gathered}
\lambda^{\circ} \backslash\{0\} \subset \operatorname{Int}\left(\gamma^{\circ}\right), \\
\operatorname{SS}(F) \cap U_{\gamma} \subset V \times \operatorname{Int}\left(\lambda^{\circ}\right) .
\end{gathered}
$$

We will prove that $L_{\lambda}(F)$ satisfies the conclusion of the lemma as well as the isomorphism $L_{\lambda}(F) \stackrel{\sim}{\sim} F$.

(ii) By (72) and Proposition 3.17 (ii) we have

$$
\mathrm{SS}(F) \subset V \times\left(\partial \gamma^{\circ} \cup \operatorname{Int}\left(\lambda^{\circ}\right)\right) .
$$

By (52) we deduce

$$
\begin{aligned}
\operatorname{SS}\left(L_{\lambda} F\right) & \subset V \times\left(\lambda^{\circ} \cap\left(\partial \gamma^{\circ} \cup \operatorname{Int}\left(\lambda^{\circ}\right)\right)\right) \\
& =V \times\left(\operatorname{Int}\left(\lambda^{\circ}\right) \cup\{0\}\right) \\
& \subset U_{\gamma} \cup T_{V}^{*} V .
\end{aligned}
$$

(iii) It remains to see that $F \simeq L_{\lambda}(F)$. We consider the distinguished triangle $\mathbf{k}_{\lambda \backslash \gamma} \star F \rightarrow L_{\lambda} F \rightarrow L_{\gamma} F \stackrel{+1}{\rightarrow}$. We have $L_{\gamma} F \stackrel{\sim}{\longrightarrow} F$. By (73), Lemma 3.16 and (52) we have

$$
\mathrm{SS}\left(\mathbf{k}_{\lambda \backslash \gamma} \star F\right) \subset V \times\left(\left(\gamma^{\circ} \backslash \operatorname{Int}\left(\lambda^{\circ}\right)\right) \cap\left(\partial \gamma^{\circ} \cup \operatorname{Int}\left(\lambda^{\circ}\right)\right)\right) \subset Z_{\gamma},
$$

which shows that $L_{\lambda} F \rightarrow F$ is an isomorphism in $\mathrm{D}^{\mathrm{b}}\left(\mathbf{k}_{V} ; U_{\gamma}\right)$. By Proposition 3.21 we obtain $F \simeq L_{\gamma}\left(L_{\lambda} F\right)$. But $\mathbf{k}_{\gamma} \star \mathbf{k}_{\lambda} \simeq \mathbf{k}_{\lambda}$ and we get finally $F \simeq L_{\lambda} F$.

Now we extend Lemma 3.25 to the case of an arbitrary manifold $M$. We consider a finite dimensional real vector space $E=E^{\prime} \times E^{\prime \prime}$ with $E^{\prime}=\mathbb{R}^{d}$. We write $x=\left(x^{\prime}, x^{\prime \prime}\right) \in E^{\prime} \times E^{\prime \prime}$ and $x^{\prime}=\left(x_{1}^{\prime}, \ldots, x_{d}^{\prime}\right) \in \mathbb{R}^{d}$. We set $U=]-1,1\left[{ }^{d} \times E^{\prime \prime}\right.$. We choose a diffeomorphism $\left.\varphi:\right]-1,1[\stackrel{\sim}{\longrightarrow}$ such that $d \varphi(t) \geq 1$ for all $t \in]-1,1[$ and we define

$$
\Phi: U \stackrel{\sim}{\longrightarrow} E, \quad \Phi\left(x_{1}^{\prime}, \ldots, x_{d}^{\prime}, x^{\prime \prime}\right)=\left(\varphi\left(x_{1}^{\prime}\right), \ldots, \varphi\left(x_{d}^{\prime}\right), x^{\prime \prime}\right) .
$$


Lemma 3.26. In the preceding situation, consider two closed convex proper cones $\gamma_{0} \subset E^{\prime \prime}$ and $C_{1} \subset E^{*}$ such that $C_{1} \subset\left(E^{* *} \times \operatorname{Int}\left(\gamma_{0}^{\circ}\right)\right) \cup\{(0,0)\}$. Then there exists another closed convex proper cone $C_{2} \subset E^{*}$ such that $C_{2} \subset$ $\left(E^{\prime *} \times \operatorname{Int}\left(\gamma_{0}^{\circ}\right)\right) \cup\{(0,0)\}$ and

$$
\Phi_{\pi} \Phi_{d}^{-1}\left(U \times C_{1}\right) \subset E \times C_{2} .
$$

Proof. (i) We assume that $\operatorname{Int}\left(\gamma_{0}^{\circ}\right)$ is non empty (otherwise the lemma is trivial). Then a closed cone of $E^{*}$ is contained in $\left(E^{* *} \times \operatorname{Int}\left(\gamma_{0}^{\circ}\right)\right) \cup\{(0,0)\}$ if and only if it is contained in $C_{a, D}:=\mathbb{R}_{\geq 0} \cdot\left([-a, a]^{d} \times D\right)$ for some $a>0$ and some compact subset $D \subset \operatorname{Int}\left(\gamma_{0}^{\circ}\right)$. Hence we may assume $C_{1}=C_{a, D}$.

(ii) Denote by $\left(x^{\prime} ; \xi^{\prime}\right)$ the coordinates on $\mathbb{R}^{d} \times\left(\mathbb{R}^{d}\right)^{*}$. We may assume that $E^{\prime \prime}=\mathbb{R}^{m}$ and we denote by $\left(x^{\prime \prime} ; \xi^{\prime \prime}\right)$ the coordinates on $E^{\prime \prime} \times\left(E^{\prime \prime}\right)^{*}$. The change of coordinates $\Phi$ defined by $y_{i}^{\prime}=\varphi\left(x_{i}^{\prime}\right)(i=1, \ldots, d), y^{\prime \prime}=x^{\prime \prime}$ associates the coordinates $(y ; \eta)=\left(y^{\prime}, y^{\prime \prime} ; \eta^{\prime}, \eta^{\prime \prime}\right)$ to the coordinates $\left(x_{1}^{\prime}, \ldots, x_{d}^{\prime}, x^{\prime \prime}\right.$; $\left.\xi_{1}^{\prime}, \ldots, \xi_{d}^{\prime}, \xi^{\prime \prime}\right)$ with

$$
\begin{aligned}
& y_{i}^{\prime}=\varphi\left(x_{i}^{\prime}\right), \quad \eta_{i}^{\prime}=d \varphi^{-1}\left(x_{i}^{\prime}\right) \cdot \xi_{i}^{\prime}, \quad(i=1, \ldots, d), \\
& y^{\prime \prime}=x^{\prime \prime}, \quad \eta^{\prime \prime}=\xi^{\prime \prime} .
\end{aligned}
$$

Since $d \varphi(t) \geq 1$, we get that $\Phi_{\pi} \Phi_{d}^{-1}\left(U \times C_{a, D}\right) \subset E \times C_{a, D}$ and we may choose $C_{2}=C_{a, D}$.

Theorem 3.27. Let $F \in \mathrm{D}_{Z_{\gamma}}^{\mathrm{b}}\left(\mathbf{k}_{E}\right)^{\perp, l}$. We assume that there exists $A \subset$ $T^{*} M \times V^{*}$ such that

(i) A is a closed strict $\gamma$-cone (see Definition 3.1),

(ii) $\mathrm{SS}(F) \cap U_{\gamma} \subset \widehat{\pi}_{E}^{-1}(A)$.

Then $\mathrm{SS}(F) \subset\left(\mathrm{SS}(F) \cap U_{\gamma}\right) \cup T_{E}^{*} E$.

Proof. Since the statement is local on $M$ we may assume that $M$ is an open subset of a vector space $W$. Then $A$ is a closed subset of $M \times W^{*} \times V^{*}$. For any $x \in M, A_{x}:=A \cap\left(\{x\} \times W^{*} \times V^{*}\right)$ is a cone satisfying

$$
A_{x} \subset\left(W^{*} \times \operatorname{Int}\left(\gamma_{0}^{\circ}\right)\right) \cup\{(0,0)\} .
$$

For $x_{0} \in M$ and for a given compact neighborhood $C$ of $x_{0}$ we may assume that there exists a closed convex cone $B$ of $W^{*} \times V^{*}$ such that $A_{x} \subset B$ for any $x \in C$ and

$$
B \subset\left(W^{*} \times \operatorname{Int}\left(\gamma_{0}^{\circ}\right)\right) \cup\{(0,0)\}
$$


We may assume $x_{0}=0 \in W$. We choose an isomorphism $W \simeq \mathbb{R}^{d}$ so that ]$-1,1\left[{ }^{d} \subset C\right.$. Then we apply a change of coordinates as in Lemma 3.26, with $E^{\prime}=W, E^{\prime \prime}=V, C_{1}=B$, and we are reduced to Lemma 3.25 applied to the vector space $W \times V$ and the cone $\gamma=\{0\} \times \gamma_{0}$.

\section{A separation theorem}

The next result is a slight generalization of Tamarkin's Theorem [12, Th. 3.2]. In this statement and its proof, we write $\widehat{\pi}$ instead of $\widehat{\pi}_{E}$ for short.

Theorem 3.28. (The separation theorem.) Let $A, B$ be two closed strict $\gamma$-cones in $T^{*} M \times V^{*}$. Let $F \in \mathrm{D}_{\widehat{\pi}^{-1}(A)}^{\mathrm{b}}\left(\mathbf{k}_{E} ; U_{\gamma}\right)$ and $G \in \mathrm{D}_{\widehat{\pi}^{-1}(B)}^{\mathrm{b}}\left(\mathbf{k}_{E} ; U_{\gamma}\right)$. Assume that $A \cap B \subset T_{M}^{*} M \times\{0\}$ and that the projection $q_{2}: M \times V \rightarrow V$ is proper on the set $\left\{\left(x, v_{1}-v_{2}\right) ;\left(x, v_{1}\right) \in \operatorname{supp} G,\left(x, v_{2}\right) \in \operatorname{supp} F\right\}$. Then

$$
\operatorname{R}_{2 *} \mathscr{H o m}{ }^{*}\left(l_{\gamma}(F), l_{\gamma}(G)\right) \simeq 0
$$

where $l_{\gamma}$ is defined in Proposition 3.21. In particular $\operatorname{Hom}_{\mathrm{D}^{\mathrm{b}}\left(\mathbf{k}_{E} ; U_{\gamma}\right)}(F, G) \simeq 0$.

Proof. We set $L=\mathscr{H} \mathrm{Hom}^{*}\left(l_{\gamma}(F), l_{\gamma}(G)\right)$ and $L^{\prime}=\mathrm{R} q_{2 *} L$. By (62) we have $L \in \mathrm{D}_{Z_{\gamma}}^{\mathrm{b}}\left(\mathbf{k}_{E}\right)^{\perp, r}$. By adjunction between $\mathrm{R} q_{2 *}$ and $q_{2}^{-1}$ we deduce $L^{\prime} \in$ $\mathrm{D}_{Z_{\gamma_{0}}}^{\mathrm{b}}\left(\mathbf{k}_{V}\right)^{\perp, r}$. It remains to check that $\mathrm{SS}\left(L^{\prime}\right) \subset Z_{\gamma_{0}}$.

By Theorem 3.27 we have $\mathrm{SS}(F) \subset \widehat{\pi}^{-1}(A)$ and $\mathrm{SS}(G) \subset \widehat{\pi}^{-1}(B)$. Then Proposition 3.13 gives $\mathrm{SS}(L) \subset \widehat{\pi}^{-1}(A) \widehat{\star}\left(\widehat{\pi}^{-1}(B)\right)^{\alpha}$. Applying Lemma 3.7 we get

$$
\mathrm{SS}(L) \cap\left(T_{M}^{*} M \times T^{*} V\right) \subset T_{E}^{*} E .
$$

Using Lemma 3.10, the hypothesis implies that $q_{2}$ is proper on $\operatorname{supp} L$. We deduce $\operatorname{SS}\left(L^{\prime}\right) \subset T_{V}^{*} V$ and thus $L^{\prime} \simeq 0$.

Proposition 3.21 and Lemma 3.18 give the first two isomorphisms in the sequence

$$
\begin{aligned}
\operatorname{Hom}_{\mathrm{D}^{\mathrm{b}}\left(\mathbf{k}_{E} ; U_{\gamma}\right)}(F, G) \simeq \operatorname{Hom}_{\mathrm{D}^{\mathrm{b}}\left(\mathbf{k}_{E}\right)}(F, G) \\
\quad \simeq \operatorname{Hom}_{\mathrm{D}^{\mathrm{b}}\left(\mathbf{k}_{E}\right)}\left(\mathbf{k}_{\gamma}, L\right) \simeq \operatorname{Hom}_{\mathrm{D}^{\mathrm{b}\left(\mathbf{k}_{V}\right)}}\left(\mathbf{k}_{\gamma_{0}}, L^{\prime}\right) \simeq 0
\end{aligned}
$$

which proves the last assertion. 


\section{Kernels}

We consider $E=M \times V, \gamma=M \times \gamma_{0}$ and a kernel $K \in \mathrm{D}^{\mathrm{b}}\left(\mathbf{k}_{E \times E}\right)$. We introduce the coordinates $\left(x, y, x^{\prime}, y^{\prime} ; \xi, \eta, \xi^{\prime}, \eta^{\prime}\right)$ on $T^{*}(E \times E)$ and we make the following hypothesis

$$
\mathrm{SS}(K) \subset\left\{\eta+\eta^{\prime}=0\right\}
$$

We recall that $L_{\gamma} \simeq \mathbf{k}_{\gamma^{+}} \circ$, where $\gamma^{+} \subset E \times E$ is defined in (55).

Proposition 3.29. Let $K \in \mathrm{D}^{\mathrm{b}}\left(\mathbf{k}_{E \times E}\right)$ which satisfies (174). Then $K \circ \mathbf{k}_{\gamma^{+}} \simeq$ $\mathbf{k}_{\gamma^{+}} \circ K$. In particular $K \circ \cdot$ sends $\mathrm{D}^{\mathrm{b}}\left(\mathbf{k}_{M}^{\gamma, l}\right)$ into itself. Moreover $\mathrm{SS}(K) \stackrel{a}{\circ}\{\eta<$ $0\} \subset\{\eta<0\}$ and $\operatorname{SS}(K) \stackrel{a}{\circ}\{\eta \geq 0\} \subset\{\eta \geq 0\}$.

Proof. We define the projection $\sigma: M \times V \times M \times V \rightarrow M \times M \times V$ as the product of $\operatorname{id}_{M \times M}$ with $\sigma_{0}: V \times V \rightarrow V,\left(y, y^{\prime}\right) \mapsto y-y^{\prime}$. Then the hypothesis (74) and Corollary 1.8 give $K \simeq \sigma^{-1}\left(K^{\prime}\right)$, where $K^{\prime}=\mathrm{R} \sigma_{*}(K)$. We also have by definition $\mathbf{k}_{\gamma^{+}} \simeq \sigma^{-1}\left(\mathbf{k}_{M \times M \times \gamma_{0}}\right)$. The base change formula applied to the Cartesian square

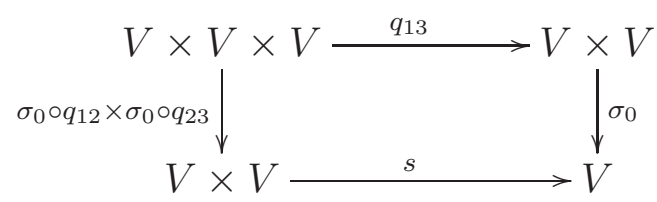

gives the first and third isomorphisms below:

$$
K \circ \mathbf{k}_{\gamma^{+}} \simeq \sigma^{-1}\left(K^{\prime} \star \mathbf{k}_{M \times M \times \gamma_{0}}\right) \simeq \sigma^{-1}\left(\mathbf{k}_{M \times M \times \gamma_{0}} \star K^{\prime}\right) \simeq \mathbf{k}_{\gamma^{+}} \circ K .
$$

The last assertion follows from the hypothesis (74).

\section{The Tamarkin category}

We particularize the preceding results to the case where $V=\mathbb{R}$ and $\gamma_{0}=$ $\{t \in \mathbb{R} ; t \geq 0\}$. Hence, with the notations of (157), we have $U_{\gamma}=\{\tau>0\}$. As in Section 2 we denote by $T_{\{\tau>0\}}^{*}(M \times \mathbb{R})$ the open subset $\{\tau>0\}$ of $T^{*}(M \times \mathbb{R})$ and we define the map

$$
\rho: T_{\{\tau>0\}}^{*}(M \times \mathbb{R}) \rightarrow T^{*} M, \quad(x, t ; \xi, \tau) \mapsto(x ; \xi / \tau) .
$$


We also use Notations 3.22. Moreover, for a closed subset $A$ of $T^{*} M$ we set

$$
\mathrm{D}_{A}^{\mathrm{b}}\left(\mathbf{k}_{M}^{\gamma}\right):=\mathrm{D}_{\rho^{-1}(A)}^{\mathrm{b}}\left(\mathbf{k}_{M \times \mathbb{R}} ;\{\tau>0\}\right) .
$$

Lemma 4.1. Let $A \subset T^{*} M$ and $F \in \mathrm{D}_{A}^{\mathrm{b}}\left(\mathbf{k}_{M}^{\gamma}\right)$. Let $A^{\prime} \subset T^{*} M \times \mathbb{R}$ be given by $A^{\prime}=\{(x ; \xi, \tau) ; \tau>0,(x ; \xi / \tau) \in A\}$ and consider $F$ as an object of $\mathrm{D}^{\mathrm{b}}\left(\mathbf{k}_{M}^{\gamma, l}\right)$. Assume that $\pi_{M}$ is proper on $A$. Then $\overline{A^{\prime}}$ is a strict $\gamma$-cone and $\mathrm{SS}(F) \subset \widehat{\pi}^{-1}\left(\overline{A^{\prime}}\right)$. In particular $\operatorname{supp}(F) \subset \pi_{M}(A) \times \mathbb{R}$.

Proof. The properness hypothesis gives $\overline{A^{\prime}}=A^{\prime} \cup\left(\pi_{M}(A) \times\{\tau=0\}\right)$ and this implies the first assertion. Then Theorem 3.27 gives $\operatorname{SS}(F) \subset$ $\widehat{\pi}^{-1}\left(\overline{A^{\prime}}\right) \cup T_{M \times \mathbb{R}}^{*}(M \times \mathbb{R})$. Hence, if $(x, t ; 0,0) \notin \widehat{\pi}^{-1}\left(\overline{A^{\prime}}\right)$, we have $\operatorname{SS}\left(\left.F\right|_{U \times \mathbb{R}}\right) \subset$ $T_{U \times \mathbb{R}}^{*}(U \times \mathbb{R})$ for some neighborhood $U$ of $x$. But $L_{\gamma} F \simeq F$ and we deduce $\left.F\right|_{U \times \mathbb{R}}=0$, which proves $(x, t ; 0,0) \notin \mathrm{SS}(F)$. So we get $\operatorname{SS}(F) \subset \widehat{\pi}^{-1}\left(\overline{A^{\prime}}\right)$.

Example 4.2. (i) Let $M=\mathbb{R}$ endowed with the coordinate $x$ and consider the set

$$
Z=\left\{(x, t) \in M \times \mathbb{R} ;-1 \leq x \leq 1,0 \leq 2 t<-x^{2}+1\right\} .
$$

Consider the sheaf $\mathbf{k}_{Z}$ and denote by $(x, t ; \xi, \tau)$ the coordinates on $T^{*}(M \times \mathbb{R})$. The set $\mathrm{SS}\left(\mathbf{k}_{Z}\right)$ is given by

$$
\begin{gathered}
\{t=0,-1 \leq x \leq 1, \tau>0, \xi=0\} \cup\left\{2 t=-x^{2}+1, \xi=x \tau, \tau>0\right\} \\
\cup\{x=-1, t=0,0 \leq-\xi \leq \tau, \tau>0\} \cup\{x=1, t=0,0 \leq \xi \leq \tau, \tau>0\} \\
\cup \bar{Z} \times\{\xi=\tau=0\} .
\end{gathered}
$$

It follows that, denoting by $(x ; u=\xi / \tau)$ the coordinates in $T^{*} M, \rho\left(\operatorname{SS}\left(\mathbf{k}_{Z}\right) \cap\right.$ $\left.\left(T^{*} M \times \dot{T}^{*} \mathbb{R}\right)\right)$ is the set

$$
\begin{aligned}
& \{u=0,-1 \leq x \leq 1\} \cup\{u=x,-1 \leq x \leq 1\} \\
& \cup\{x=-1,-1 \leq u \leq 0\} \cup\{x=1,0 \leq u \leq 1\} .
\end{aligned}
$$

(ii) Let $a \in \mathbb{R}$ and consider the set $Z=\{(x, t) \in M \times \mathbb{R} ; t \geq a x\}$. Then $\rho\left(\mathrm{SS}\left(\mathbf{k}_{Z}\right)\right)$ in $T^{*} M$ is the set $\{(x ; u) ; u=a\}$.

(iii) If $G$ is a sheaf on $M$ and $F=G \otimes \mathbf{k}_{s \geq 0}$, then $\rho(\operatorname{SS}(F))=\operatorname{SS}(G)$.

\section{The separation theorem}

Using Lemma 4.1 we get the following particular case of Theorem 3.28

Theorem 4.3. (see [12, Th. 3.2].) Let $A$ and $B$ be two compact subsets of $T^{*} M$ and assume that $A \cap B=\emptyset$. Then, for any $F \in \mathrm{D}_{A}^{\mathrm{b}}\left(\mathbf{k}_{M}^{\gamma}\right)$ and $G \in \mathrm{D}_{B}^{\mathrm{b}}\left(\mathbf{k}_{M}^{\gamma}\right)$, we have $\operatorname{Hom}_{\mathrm{D}^{\mathrm{b}}\left(\mathbf{k}_{M}^{\gamma}\right)}(F, G) \simeq 0$. 


\section{$5 \quad$ Localization by torsion objects}

In [12], Tamarkin introduces the notion of torsion objects, but does not study the category of such objects systematically. Hence, most of the results of this section are new.

In this section we set for short $Z=\left(T^{*} M\right) \times \mathbb{R} \times\{\tau \geq 0\}$, a closed subset of $T^{*}(M \times \mathbb{R})$. Recall that $\mathrm{D}_{Z}^{\mathrm{b}}\left(\mathbf{k}_{M \times \mathbb{R}}\right)$ is the subcategory of $F \in \mathrm{D}^{\mathrm{b}}\left(\mathbf{k}_{M \times \mathbb{R}}\right)$ such that $\operatorname{SS}(F) \subset Z$. By Proposition 3.9 we have $F \in \mathrm{D}_{Z}^{\mathrm{b}}\left(\mathbf{k}_{M \times \mathbb{R}}\right)$ if and only if the morphism (43) is an isomorphism, which reads

$$
F \star_{n p} \mathbf{k}_{M \times[0,+\infty[} \stackrel{\sim}{\longrightarrow} F
$$

Define the map

$$
T_{c}: M \times \mathbb{R} \rightarrow M \times \mathbb{R}, \quad(x, t) \mapsto(x, t+c) .
$$

For $F \in \mathrm{D}_{Z}^{\mathrm{b}}\left(\mathbf{k}_{M \times \mathbb{R}}\right)$ we deduce easily from (76)

$$
F \star_{n p} \mathbf{k}_{M \times[c,+\infty[} \stackrel{\sim}{\longrightarrow} T_{c *} F .
$$

The inclusions $[d,+\infty[\subset[c,+\infty[$, for $c \leq d$, induce natural morphisms of functors from $\mathrm{D}_{Z}^{\mathrm{b}}\left(\mathbf{k}_{M \times \mathbb{R}}\right)$ to itself

$$
\tau_{c, d}: T_{c *} \rightarrow T_{d *}, \quad c \leq d
$$

We have the identities:

$$
\begin{aligned}
& T_{c *} \circ T_{d *} \simeq T_{(c+d)_{*}}, \quad c, d \in \mathbb{R}, \\
& T_{e *}\left(\tau_{c, d}(\bullet)\right)=\tau_{e+c, e+d}(\bullet)=\tau_{c, d}\left(T_{e *}(\bullet)\right), \quad c \leq d, e \in \mathbb{R}, \\
& \tau_{c, d} \circ \tau_{d, e}=\tau_{c, e}, \quad c \leq d \leq e .
\end{aligned}
$$

Definition 5.1. (Tamarkin.) An object $F \in \mathrm{D}_{Z}^{\mathrm{b}}\left(\mathbf{k}_{M \times \mathbb{R}}\right)$ is called a torsion object if $\tau_{0, c}(F)=0$ for some $c \geq 0$ (and hence all $c^{\prime} \geq c$ ).

Let $F \in \mathrm{D}_{Z}^{\mathrm{b}}\left(\mathbf{k}_{M \times \mathbb{R}}\right)$ and assume that $F$ is supported by $M \times[a, b]$ for some compact interval $[a, b]$ of $\mathbb{R}$. Then $F$ is a torsion object.

Remark 5.2. One can give an alternative definition of the torsion objects by using the classical notion of ind-objects (see [6] for an exposition). An object $F \in \mathrm{D}_{Z}^{\mathrm{b}}\left(\mathbf{k}_{M \times \mathbb{R}}\right)$ is torsion if and only if the natural morphism $F \rightarrow \underset{c}{\stackrel{\text { "limp }}{\longrightarrow}} T_{c *} F$
is the zero morphism. 
We let $\mathscr{N}_{\text {tor }}$ be the full subcategory of $\mathrm{D}_{Z}^{\mathrm{b}}\left(\mathbf{k}_{M \times \mathbb{R}}\right)$ consisting of torsion objects.

Lemma 5.3. Let $F \stackrel{u}{\rightarrow} G \stackrel{v}{\rightarrow} H \stackrel{w}{\rightarrow} F[1]$ be a distinguished triangle in $\mathrm{D}_{Z}^{\mathrm{b}}\left(\mathbf{k}_{M \times \mathbb{R}}\right)$.

(i) If $H$ belongs to $\mathscr{N}_{\text {tor }}$, then there exist $c \geq 0$ and $\alpha: G \rightarrow T_{c *} F$ such that $\tau_{0, c}(F)=\alpha \circ u$.

(ii) If there exist $c \geq 0$ and $\alpha: G \rightarrow T_{c *} F$ making the diagram

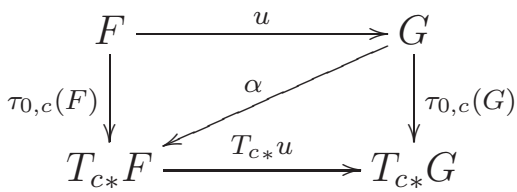

commutative, then $H \in \mathscr{N}_{\text {tor }}$.

Proof. (i) Choose $c \geq 0$ such that $\tau_{0, c}(H) \simeq 0$ and consider the diagram with solid arrows

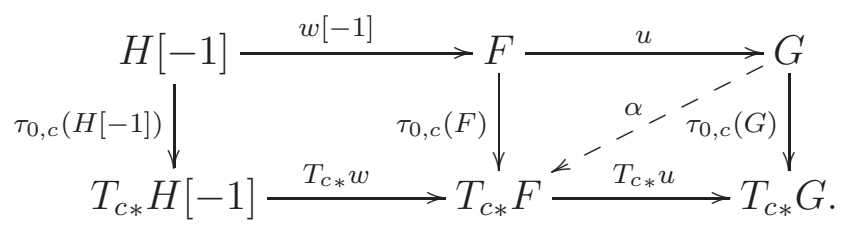

Since $\tau_{0, c}(H[-1]) \simeq 0$, we have $\tau_{0, c}(F) \circ w[-1]=0$. Since $\operatorname{Hom}\left(\bullet, T_{c *} F\right)$ is a cohomological functor we deduce the existence of $\alpha$.

(ii) We apply $T_{c *}$ twice and obtain morphisms of distinguished triangles:

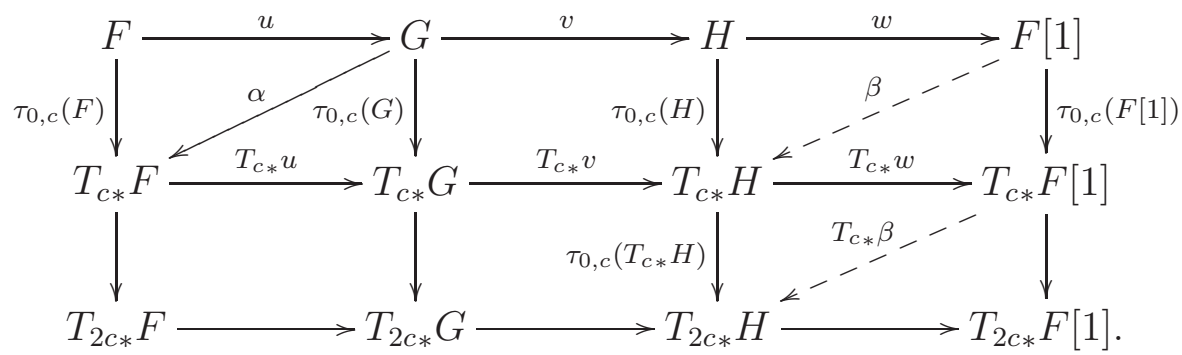

By hypothesis $\tau_{0, c}(H) \circ v=T_{c *} v \circ T_{c *} u \circ \alpha=0$. As above, we deduce the existence of $\beta$ such that $\tau_{0, c}(H)=\beta \circ w$. Applying the morphism of functors $\tau_{0, c}:$ id $\rightarrow T_{c}$ to $\beta$ we find

$$
\tau_{0, c}\left(T_{c *} H\right) \circ \beta=T_{c *} \beta \circ \tau_{0, c}(F[1]) .
$$


We deduce:

$$
\begin{aligned}
\tau_{0, c}\left(T_{c *} H\right) \circ \tau_{0, c}(H)=\tau_{0, c}\left(T_{c *} H\right) \circ \beta \circ w= & T_{c *} \beta \circ \tau_{0, c}(F[1]) \circ w \\
& =T_{c *} \beta \circ \alpha[1] \circ u[1] \circ w=0 .
\end{aligned}
$$

Using (78) we obtain $\tau_{0,2 c}(H) \simeq 0$ so that $H \in \mathscr{N}_{\text {tor }}$.

Theorem 5.4. The subcategory $\mathscr{N}_{\text {tor }}$ is a null system in $\mathrm{D}_{Z}^{\mathrm{b}}\left(\mathbf{k}_{M \times \mathbb{R}}\right)$.

Proof. It is clear that an object isomorphic to a torsion object is itself a torsion object and that $\mathscr{N}_{\text {tor }}$ is stable by the shift functor. Hence it remains to check that if $F \rightarrow G \rightarrow H \stackrel{+1}{\longrightarrow}$ is a distinguished triangle with $F, G \in \mathscr{N}_{\text {tor }}$ then $H \in \mathscr{N}_{\text {tor. }}$. We choose $c \geq 0$ such that $\tau_{0, c}(F)=0$ and $\tau_{0, c}(G)=0$ and we apply Lemma 5.3 (ii) to the diagram

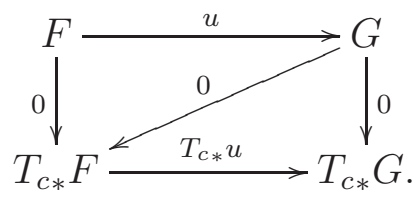

Corollary 5.5. For any $F \in \mathrm{D}_{Z}^{\mathrm{b}}\left(\mathbf{k}_{M \times \mathbb{R}}\right)$ and any $c \geq 0$, the cone of $\tau_{0, c}(F)$ is a torsion object.

Proof. We apply Lemma 5.3 (ii) to the commutative diagram

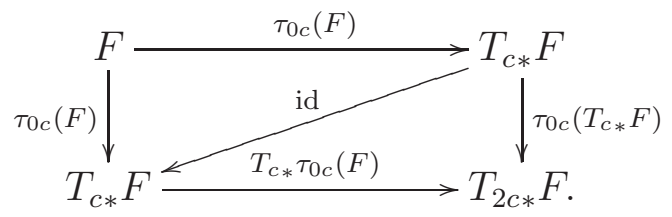

The subcategory $\mathrm{D}^{\mathrm{b}}\left(\mathbf{k}_{M}^{\gamma, l}\right)$ of $\mathrm{D}^{\mathrm{b}}\left(\mathbf{k}_{M \times \mathbb{R}}\right)$ is contained in $\mathrm{D}_{Z}^{\mathrm{b}}\left(\mathbf{k}_{M \times \mathbb{R}}\right)$. So we can define torsion objects in $\mathrm{D}^{\mathrm{b}}\left(\mathbf{k}_{M}^{\gamma, l}\right)$ or in the equivalent category $\mathrm{D}^{\mathrm{b}}\left(\mathbf{k}_{M}^{\gamma}\right)$. We let $\mathscr{N}_{\text {tor }}^{\gamma}$ be the subcategory of torsion objects in $\mathrm{D}^{\mathrm{b}}\left(\mathbf{k}_{M}^{\gamma}\right)$. Then Theorem 5.4 implies that $\mathscr{N}_{\text {tor }}^{\gamma}$ is a null system.

Definition 5.6. The triangulated category $\mathscr{T}\left(\mathbf{k}_{M}\right)$ is the localization of $\mathrm{D}^{\mathrm{b}}\left(\mathbf{k}_{M}^{\gamma}\right)$ by the null system $\mathscr{N}_{\text {tor }}^{\gamma}$. In other words, $\mathscr{T}\left(\mathbf{k}_{M}\right)=\mathrm{D}^{\mathrm{b}}\left(\mathbf{k}_{M}^{\gamma}\right) / \mathscr{N}_{\text {tor }}^{\gamma}$. 
By Corollary [5.5, $\tau_{0, c}(G)$ becomes invertible in $\mathscr{T}\left(\mathbf{k}_{M}\right)$ for any $G \in$ $\mathrm{D}^{\mathrm{b}}\left(\mathbf{k}_{M}^{\gamma}\right)$. Hence for a morphism $u: F \rightarrow G$ in $\mathrm{D}^{\mathrm{b}}\left(\mathbf{k}_{M}^{\gamma}\right)$ and for $c \geq 0$ we can define $\tau_{0, c}(G)^{-1} \circ u: F \rightarrow G$ in $\mathscr{T}\left(\mathbf{k}_{M}\right)$. The family of $\tau_{c, c^{\prime}}(G)^{\prime}$ 's defines an inductive system $\left\{T_{c *} G\right\}_{c}$ and we have $\tau_{0, c^{\prime}}(G)^{-1} \circ \tau_{c, c^{\prime}}(G) \circ u=\tau_{0, c}(G)^{-1} \circ u$ for $c^{\prime} \geq c$. This defines a natural morphism:

$$
\lim _{c \rightarrow+\infty} \operatorname{Hom}_{\mathrm{D}^{\mathrm{b}}\left(\mathbf{k}_{M}^{\gamma}\right)}\left(F, T_{c *} G\right) \rightarrow \operatorname{Hom}_{\mathscr{T}\left(\mathbf{k}_{M}\right)}(F, G)
$$

Proposition 5.7. For any $F, G \in \mathrm{D}^{\mathrm{b}}\left(\mathbf{k}_{M}^{\gamma}\right)$ the morphism (81) is an isomorphism.

Proof. (i) Let us first show that (81) is surjective. A morphism $u: F \rightarrow G$ in $\mathscr{T}\left(\mathbf{k}_{M}\right)$ is given by $F \stackrel{v}{\rightarrow} G^{\prime} \stackrel{s}{\leftarrow} G$, where the cone of $s$ is a torsion object. By Lemma 5.3 (i) there exist $c \geq 0$ and $\alpha: G^{\prime} \rightarrow T_{c *} G$ such that $\tau_{0, c}(G)=\alpha \circ s$ :

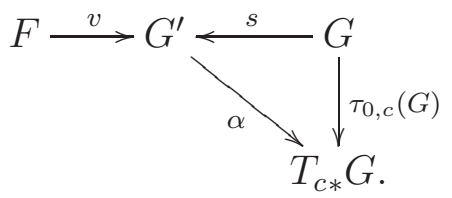

Hence we obtain $u=\tau_{0, c}(G)^{-1} \circ \alpha \circ v$ in $\mathscr{T}\left(\mathbf{k}_{M}\right)$. In other words $u$ is the image of $\alpha \circ v$ by (81).

(ii) Now we show that (81) is injective. We consider $u: F \rightarrow T_{c *} G$ in $\mathrm{D}^{\mathrm{b}}\left(\mathbf{k}_{M}^{\gamma}\right)$ such that $\tau_{0 c}(G)^{-1} \circ u=0$ in $\mathscr{T}\left(\mathbf{k}_{M}\right)$. Then $u=0$ in $\mathscr{T}\left(\mathbf{k}_{M}\right)$ and this means that there exists $s: T_{c *} G \rightarrow G^{\prime}$ such that the cone of $s$ is a torsion object and $s \circ u=0$ in $\mathrm{D}^{\mathrm{b}}\left(\mathbf{k}_{M}^{\gamma}\right)$. By Lemma 5.3 (i) there exist $d \geq 0$ and $\alpha: G^{\prime} \rightarrow T_{(c+d)_{*}} G$ such that $\tau_{c, c+d}(G)=\alpha \circ s:$

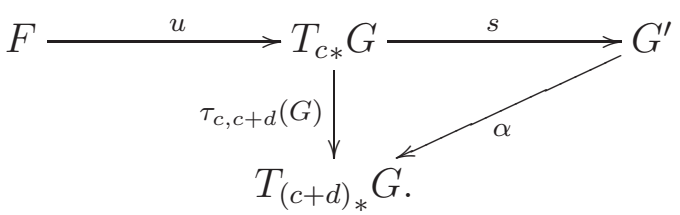

We obtain $\tau_{c, c+d}(G) \circ u=\alpha \circ s \circ u=0$ which means that the image of $u$ in the left hand side of (81) is zero, as required.

Recall the functor $\Psi_{\gamma}$ in (69). 
Corollary 5.8. The composition $\mathrm{D}^{\mathrm{b}}\left(\mathbf{k}_{M}\right) \stackrel{\Psi_{\gamma}}{\longrightarrow} \mathrm{D}^{\mathrm{b}}\left(\mathbf{k}_{M \times \mathbb{R}} ; U_{\gamma}\right) \rightarrow \mathscr{T}\left(\mathbf{k}_{M}\right)$ is a fully faithful functor.

Proof. For $F, G \in \mathrm{D}^{\mathrm{b}}\left(\mathbf{k}_{M}\right)$, the proof of Proposition 3.24 gives as well

$$
\operatorname{Hom}_{\mathrm{D}^{\mathrm{b}}\left(\mathbf{k}_{M}\right)}(G, F) \stackrel{\sim}{\longrightarrow} \operatorname{Hom}_{\mathrm{D}^{\mathrm{b}}\left(\mathbf{k}_{M \times \mathbb{R}}\right)}\left(G \otimes \mathbf{k}_{[0,+\infty[}, F \otimes \mathbf{k}_{[c,+\infty[}\right)
$$

for any $c \geq 0$. Then the result follows from Proposition 5.7 .

\section{Strict cones and torsion}

For a connected manifold $M$ and $F \in \mathrm{D}_{Z}^{\mathrm{b}}\left(\mathbf{k}_{M \times \mathbb{R}}\right)$ we give a condition on $\mathrm{SS}(F)$ which implies that $F$ is torsion over any compact subset as soon as it is torsion at one point.

We first give a preliminary result on $M \times I \times \mathbb{R}$. We set $E=\mathbb{R}^{2}$ and we take coordinates $(s, t ; \sigma, \tau)$ on $T^{*} E$. We fix $\alpha>0$ and define the cone $\gamma_{\alpha}=$ $\{(s, t) ; t \geq \alpha|s|\}$ in $E$. We set $U_{\alpha}=E \times \operatorname{Int} \gamma_{\alpha}^{\circ}$. We recall Proposition 3.9, reformulated using (44): for $F \in \mathrm{D}^{\mathrm{b}}\left(\mathbf{k}_{M \times E}\right)$, we have $\operatorname{SS}(F) \subset T^{*} M \times \bar{U}_{\alpha}$ if and only if

$$
F \star_{n p} \mathbf{k}_{M \times \gamma_{\alpha}} \simeq \mathrm{R}_{E *} \mathrm{R} \Gamma_{M \times E \times \operatorname{Int} \gamma_{\alpha}}\left(q_{1}^{-1} F\right) \stackrel{\sim}{\longrightarrow} F,
$$

where $s_{E}: M \times E \times E \rightarrow M \times E$ is the sum of $E$.

Proposition 5.9. Let $I$ be an interval of $\mathbb{R}, M$ a manifold and $q: M \times I \times$ $\mathbb{R} \rightarrow M \times \mathbb{R}$ the projection. Set $\gamma=I \times\left[0,+\infty\left[\right.\right.$. Let $F \in \mathrm{D}^{\mathrm{b}}\left(\mathbf{k}_{M \times I \times \mathbb{R}}\right)$. We assume that there exists a closed strict $\gamma$-cone $A \subset\left(T^{*} I\right) \times \mathbb{R}$ such that $\mathrm{SS}(F) \subset T^{*} M \times \widehat{\pi}^{-1}(A)$. Then, for any $s_{1}<s_{2} \in I, \mathrm{R}_{*}\left(F \otimes \mathbf{k}_{M \times\left[s_{1}, s_{2}[\times \mathbb{R}\right.}\right)$ and $\mathrm{R} q_{*}\left(F \otimes \mathbf{k}_{\left.M \times] s_{1}, s_{2}\right] \times \mathbb{R}}\right)$ are torsion objects of $\mathrm{D}_{Z}^{\mathrm{b}}\left(\mathbf{k}_{M \times \mathbb{R}}\right)$.

Proof. (i) We only consider $G:=\mathrm{R} q_{*}\left(F \otimes \mathbf{k}_{M \times\left[s_{1}, s_{2}[\times \mathbb{R}\right.}\right)$, the other case being similar. We may restrict ourselves to a relatively compact subinterval of $I$ containing $s_{1}$ and $s_{2}$. Hence we may assume that $\mathrm{SS}(F)$ is contained in $T^{*} M \times\{\tau \geq a|\sigma|\}$ for some $a>0$. Then, applying Lemma 3.26 and changing $a$ if necessary, we may assume that $I=\mathbb{R}$.

(ii) We set $\alpha=a^{-1}$ so that $\gamma_{\alpha}^{\circ}=\{\tau \geq a|\sigma|\}$ and $\operatorname{SS}(F) \subset T^{*} M \times \bar{U}_{\alpha}$. Since $\mathrm{SS}\left(\mathbf{k}_{M \times\left[s_{1}, s_{2}[\times \mathbb{R}\right.}\right) \subset T_{M}^{*} M \times T^{*} \mathbb{R} \times T_{\mathbb{R}}^{*} \mathbb{R}$, Corollary 1.7 gives $F \otimes \mathbf{k}_{M \times\left[s_{1}, s_{2}[\times \mathbb{R}\right.} \simeq$ $\mathrm{R} \Gamma_{\left.M \times] s_{1}, s_{2}\right] \times \mathbb{R}}(F)$ and the formula (182) gives

$$
G \simeq \mathrm{R} q_{*} \mathrm{R} s_{E *} \mathrm{R} \Gamma_{M \times D}\left(q_{1}^{-1} F\right),
$$


where $D=\left(E \times \operatorname{Int} \gamma_{\alpha}\right) \cap\left\{\left(s, t, s^{\prime}, t^{\prime}\right) ; s_{1}<s+s^{\prime} \leq s_{2}\right\}$. We consider the commutative diagram

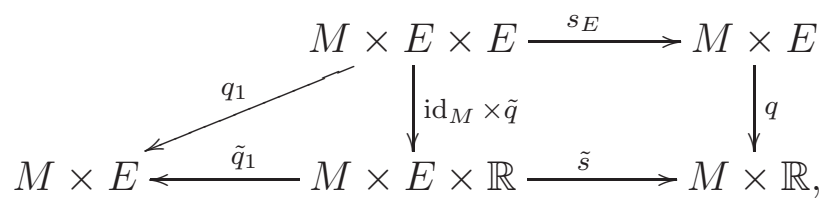

where $\tilde{q}\left(s, t, s^{\prime}, t^{\prime}\right)=\left(s, t, t^{\prime}\right), \tilde{q}_{1}\left(x, s, t, t^{\prime}\right)=(x, s, t)$ and $\tilde{s}\left(x, s, t, t^{\prime}\right)=(x, t+$ $\left.t^{\prime}\right)$. The adjunction between $\mathrm{R}\left(\operatorname{id}_{M} \times \tilde{q}\right)$ ! and $\left(\operatorname{id}_{M} \times \tilde{q}\right)$ ! gives

$$
\begin{aligned}
G & \simeq \operatorname{R} \tilde{s}_{*} \operatorname{R}\left(\operatorname{id}_{M} \times \tilde{q}\right)_{*} \operatorname{R} \mathscr{H} o m\left(\mathbf{k}_{M \times D},\left(\operatorname{id}_{M} \times \tilde{q}\right)^{!} \tilde{q}_{1}^{-1} F\right)[-1] \\
& \simeq \operatorname{R} \tilde{s}_{*} \operatorname{R} \mathscr{H} o m\left(\mathbf{k}_{M} \otimes \operatorname{R} \tilde{q}_{!} \mathbf{k}_{D}, \tilde{q}_{1}^{-1} F\right)[-1] .
\end{aligned}
$$

(iii) Through the isomorphism (82) the morphism $\tau_{c}(F)$ is induced by the morphism $\mathbf{k}_{T_{c}\left(E \times \operatorname{Int} \gamma_{\alpha}\right)} \rightarrow \mathbf{k}_{E \times \operatorname{Int} \gamma_{\alpha}}$, where $T_{c}\left(s, t, s^{\prime}, t^{\prime}\right)=\left(s, t, s^{\prime}, t^{\prime}+c\right)$. Using (83) it follows that $\tau_{c}(G)$ is induced by the morphism $u_{c}: \mathbf{k}_{T_{c}(D)} \rightarrow \mathbf{k}_{D}$. Hence it is enough to see that the image of $u_{c}$ by $\mathrm{R} \tilde{q}_{\text {! }}$ is the zero morphism. In the remainder of the proof we show that $\mathrm{R} \tilde{q}_{!} \mathbf{k}_{D}$ and $\mathrm{R} \tilde{q}_{!} \mathbf{k}_{T_{c}(D)}$ have disjoint supports for $c$ big enough.

(iv) For a given point $\left(s, t, t^{\prime}\right) \in E \times \mathbb{R}$ we have $\tilde{q}^{-1}\left(s, t, t^{\prime}\right) \cap D=\emptyset$ if $t^{\prime}<0$ and otherwise

$$
\begin{aligned}
\tilde{q}^{-1}\left(s, t, t^{\prime}\right) \cap D & =\left\{s^{\prime} ; s_{1}-s<s^{\prime} \leq s_{2}-s, t^{\prime} \geq \alpha\left|s^{\prime}\right|\right\} \\
& \left.=] s_{1}-s, s_{2}-s\right] \cap\left[-\alpha^{-1} t^{\prime}, \alpha^{-1} t^{\prime}\right]
\end{aligned}
$$

This is $\emptyset$ or a half closed interval when $t^{\prime}$ is not in $I_{s}:=\left[-\alpha\left(s_{2}-s\right),-\alpha\left(s_{1}-s\right)[\right.$. It follows that $\operatorname{supp}\left(\mathrm{R} \tilde{q}_{!} \mathbf{k}_{D}\right)$ is contained in $D^{\prime}:=\left\{\left(s, t, t^{\prime}\right) ; t^{\prime} \in \overline{I_{s}}\right\}$. The support of $\mathrm{R} \tilde{q}_{!} \mathbf{k}_{T_{c}(D)}$ is contained in $T_{c}^{\prime}\left(D^{\prime}\right)$, with $T_{c}^{\prime}\left(s, t, t^{\prime}\right)=\left(s, t, c+t^{\prime}\right)$. Since $I_{s}$ is of length $\alpha\left(s_{2}-s_{1}\right)$ (independent of $s$ ) we obtain $D^{\prime} \cap T_{c}^{\prime}\left(D^{\prime}\right)=\emptyset$ for $c>\alpha\left(s_{2}-s_{1}\right)$.

From now on, we consider a connected manifold $M$ and $F \in \mathrm{D}^{\mathrm{b}}\left(\mathbf{k}_{M \times \mathbb{R}}\right)$. We set $\gamma=M \times[0,+\infty[$ and we make the hypothesis

$$
\mathrm{SS}(F) \subset \widehat{\pi}^{-1}(A) \text { for some closed } \gamma \text {-strict cone } A \subset\left(T^{*} M\right) \times \mathbb{R} .
$$

In particular $F \in \mathrm{D}_{\{\tau \geq 0\}}^{\mathrm{b}}\left(\mathbf{k}_{M \times \mathbb{R}}\right)$. 
Lemma 5.10. Let $F \in \mathrm{D}^{\mathrm{b}}\left(\mathbf{k}_{M \times \mathbb{R}}\right)$ satisfying (84). We assume that there exists $x \in M$ such that $\left.F\right|_{\{x\} \times \mathbb{R}}$ is a torsion object in $\mathrm{D}_{\{\tau \geq 0\}}^{\mathrm{b}}\left(\mathbf{k}_{\mathbb{R}}\right)$. Then there exists a neighborhood $U$ of $x$ such that $\left.F\right|_{U \times \mathbb{R}}$ is a torsion object in $\mathrm{D}_{\{\tau \geq 0\}}^{\mathrm{b}}\left(\mathbf{k}_{U \times \mathbb{R}}\right)$.

Proof. (i) We may assume that $M$ is an open set in some vector space $V$ and $x=0$. We take coordinates $(x, t ; \xi, \tau)$ on $T^{*}(M \times \mathbb{R})$. We may also assume that $\operatorname{SS}(F) \subset\{\tau \geq a\|\xi\|\}$ for some $a>0$ and that $M$ contains the open ball of radius 1 , say $B$. We set $I=]-1,1[$ and take coordinates $(s ; \sigma)$ on $T^{*} I$. We define the homotopy $h: B \times I \times \mathbb{R} \rightarrow B \times \mathbb{R},(x, s, t) \mapsto(s x, t)$. For $s_{0} \in I$ we set $h_{s_{0}}=h\left(\cdot, s_{0}, \cdot\right)$.

(ii) We check that $h^{-1}\left(\left.F\right|_{B \times \mathbb{R}}\right)$ satisfies the hypothesis of Proposition 5.9. We have $h_{\pi}(x, s, t ; \xi, \tau)=(s x ; t \xi, \tau)$ and $h_{d}(x, s, t ; \xi, \tau)=(x, s, t ; s \xi,\langle x, \xi\rangle, \tau)$. Hence Ker $h_{d}$ is contained in $\{\tau=0\}$. Since $\operatorname{SS}(F) \cap\{\tau=0\}$ is contained in the zero-section, $F$ is non-characteristic for $h$ and we find

$$
\mathrm{SS}\left(h^{-1}(F)\right) \subset\left\{\left(x^{\prime}, s^{\prime}, t^{\prime} ; \xi^{\prime}, \sigma^{\prime}, \tau^{\prime}\right) ; \sigma^{\prime}=\left\langle x^{\prime}, \xi^{\prime}\right\rangle, \tau^{\prime} \geq a\left\|\xi^{\prime}\right\| /\left|s^{\prime}\right|\right\}
$$

On $B \times I$ we have $\left|s^{\prime}\right| \leq 1$ and $\left|\left\langle x^{\prime}, \xi^{\prime}\right\rangle\right| \leq\left\|\xi^{\prime}\right\|$. We deduce $\operatorname{SS}\left(h^{-1}(F)\right) \subset$ $\left\{\tau^{\prime} \geq a\left|\sigma^{\prime}\right|\right\}$ on $B \times I \times \mathbb{R}$, as required.

(iii) We apply Proposition 5.9 to $h^{-1}(F)$ on $B \times I \times \mathbb{R}$ with $s_{1}=0, s_{2}=1 / 2$. For $J \subset I$ we set $G_{J}=\operatorname{R} q_{*}\left(h^{-1}\left(\left.F\right|_{B \times \mathbb{R}}\right) \otimes \mathbf{k}_{M \times J \times \mathbb{R}}\right)$. We note that $G_{\{s\}} \simeq$ $h_{s}^{-1}\left(\left.F\right|_{B \times \mathbb{R}}\right)$ for any $s \in I$. We have the distinguished triangles on $B \times \mathbb{R}$

$$
G_{0,1 / 2]} \rightarrow G_{[0,1 / 2]} \rightarrow G_{\{0\}} \stackrel{+1}{\longrightarrow}, \quad G_{[0,1 / 2[} \rightarrow G_{[0,1 / 2]} \rightarrow G_{\{1 / 2\}} \stackrel{+1}{\rightarrow},
$$

where $G_{0,1 / 2]}$ and $G_{[0,1 / 2[}$ are torsion by Proposition 5.9. Since $h_{0}$ is the contraction $B \times \mathbb{R} \rightarrow\{0\} \times \mathbb{R}$ the hypothesis implies that $G_{\{0\}}$ is torsion. Hence $G_{[0,1 / 2]}$ is torsion by the first distinguished triangle and then $G_{\{1 / 2\}}$ also is torsion by the second one. Since $h_{1 / 2}$ is a diffeomorphism from $B \times \mathbb{R}$ to $U \times \mathbb{R}$, where $U$ is the ball of radius $1 / 2$ we deduce that $\left.F\right|_{U \times \mathbb{R}}$ is torsion.

Lemma 5.11. Let $F \in \mathrm{D}^{\mathrm{b}}\left(\mathbf{k}_{M \times \mathbb{R}}\right)$ satisfying (84). We assume that there exists $x_{0} \in M$ such that $\left.F\right|_{\left\{x_{0}\right\} \times \mathbb{R}}$ is a torsion object in $\mathrm{D}_{\{\tau \geq 0\}}^{\mathrm{b}}\left(\mathbf{k}_{\mathbb{R}}\right)$. Then $\left.F\right|_{\{x\} \times \mathbb{R}}$ also is a torsion object in $\mathrm{D}_{\{\tau \geq 0\}}^{\mathrm{b}}\left(\mathbf{k}_{\mathbb{R}}\right)$ for all $x \in M$.

Proof. We set $I=]-1,1[$ and we choose an immersion $i: I \rightarrow M$ such that $i(0)=x_{0}$ and $i(1 / 2)=x$. Then $i^{-1} F$ satisfies the hypothesis of 
Proposition 5.9 on $I \times \mathbb{R}$. We let $q: I \times \mathbb{R} \rightarrow \mathbb{R}$ be the projection. Then $\left.F\right|_{\{i(s)\} \times \mathbb{R}} \simeq \mathrm{R} q_{*}\left(i^{-1} F \otimes \mathbf{k}_{\{s\} \times \mathbb{R}}\right)$ for any $s \in I$. Now we have the distinguished triangles

$$
\begin{array}{r}
\left.\mathrm{R} q_{*}\left(i^{-1} F \otimes \mathbf{k}_{] 0,1 / 2] \times \mathbb{R}}\right) \rightarrow \mathrm{R} q_{*}\left(i^{-1} F \otimes \mathbf{k}_{[0,1 / 2] \times \mathbb{R}}\right) \rightarrow i^{-1} F\right|_{\left\{x_{0}\right\} \times \mathbb{R}} \stackrel{+1}{\rightarrow}, \\
\left.\mathrm{R} q_{*}\left(i^{-1} F \otimes \mathbf{k}_{[0,1 / 2[\times \mathbb{R}}\right) \rightarrow \mathrm{R} q_{*}\left(i^{-1} F \otimes \mathbf{k}_{[0,1 / 2] \times \mathbb{R}}\right) \rightarrow i^{-1} F\right|_{\{x\} \times \mathbb{R}} \stackrel{+1}{\longrightarrow}
\end{array}
$$

and we conclude as in part (iii) of the proof of Lemma 5.10.

Theorem 5.12. Let $M$ be a connected manifold and let $F \in \mathrm{D}^{\mathrm{b}}\left(\mathbf{k}_{M \times \mathbb{R}}\right)$ satisfying (84). Then the following assertions are equivalent:

(i) there exists $x_{0} \in M$ such that $\left.F\right|_{\left\{x_{0}\right\} \times \mathbb{R}}$ is a torsion object in $\mathrm{D}_{\{\tau \geq 0\}}^{\mathrm{b}}\left(\mathbf{k}_{\mathbb{R}}\right)$,

(ii) for any relatively compact open subset $U \subset M$ the restriction $\left.F\right|_{U \times \mathbb{R}}$ is a torsion object in $\mathrm{D}_{\{\tau \geq 0\}}^{\mathrm{b}}\left(\mathbf{k}_{U \times \mathbb{R}}\right)$.

Proof. We only need to prove that (i) implies (ii). By Lemmas 5.10 and 5.11 we can find a finite cover of $\bar{U}$, say $\left\{U_{i}\right\}, i=1, \ldots, n$, such that $\left.F\right|_{U_{i} \times \mathbb{R}}$ is torsion. We conclude with the remark that, for any two open subsets $V, W \subset M$, if $\left.F\right|_{V \times \mathbb{R}}$ and $\left.F\right|_{W \times \mathbb{R}}$ are torsion, then so is $\left.F\right|_{(V \cup W) \times \mathbb{R} \text {. Indeed we }}$ apply Lemma 5.3 to the triangle $F_{(V \cap W) \times \mathbb{R}} \rightarrow F_{V \times \mathbb{R}} \oplus F_{W \times \mathbb{R}} \rightarrow F_{(V \cup W) \times \mathbb{R}} \stackrel{+1}{\longrightarrow}$ and the commutative square

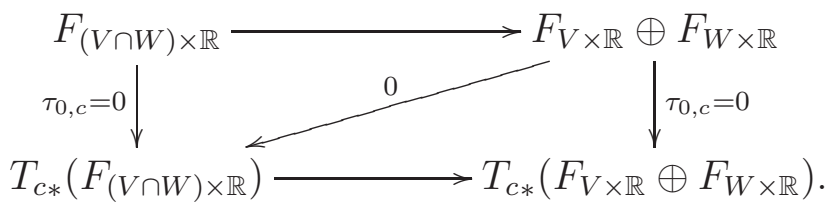

\section{Tamarkin's non displaceability theorem}

We will explain here Tamarkin's non displaceability theorem which gives a criterion in order that two compact subsets of $T^{*} M$ are non displaceable.

In this section we consider a Hamiltonian isotopy $\Phi: T^{*} M \times I \rightarrow T^{*} M$ satisfying (29), that is, there exists a compact set $C \subset T^{*} M$ such that $\left.\varphi_{s}\right|_{T^{*} M \backslash C}$ is the identity for all $s \in I$. 
Let $\widetilde{\Phi}: \dot{T}^{*}(M \times \mathbb{R}) \times I \rightarrow \dot{T}^{*}(M \times \mathbb{R})$ be the homogeneous Hamiltonian isotopy given by Proposition 2.2 and $\widetilde{\Lambda} \subset T^{*}(M \times \mathbb{R} \times M \times \mathbb{R} \times I)$ the conic Lagrangian submanifold associated to $\widetilde{\Phi}$ in (26). Let $\widetilde{K} \in \mathrm{D}^{\mathrm{lb}}\left(\mathbf{k}_{M \times \mathbb{R} \times M \times \mathbb{R} \times I}\right)$ be the quantization of $\widetilde{\Phi}$ given in Theorem 2.1 .

\section{Invariance by Hamiltonian isotopy}

For $J \subset I$ a relatively compact subinterval of $I$, we introduce the kernel

$$
K^{J}=\operatorname{R} q_{1234 !}\left(\widetilde{K} \otimes \mathbf{k}_{M \times \mathbb{R} \times M \times \mathbb{R} \times J}\right) \in \mathrm{D}^{\mathrm{b}}\left(\mathbf{k}_{M \times \mathbb{R} \times M \times \mathbb{R}}\right),
$$

where $q_{1234}$ is the projection on the first four factors. We remark that $\widetilde{K}$ and $K^{J}$ satisfy the hypothesis (74). Hence, by Proposition 3.29, composition with $K^{J}$ defines a functor

$$
\Psi_{J}: \mathrm{D}^{\mathrm{b}}\left(\mathbf{k}_{M}^{\gamma}\right) \rightarrow \mathrm{D}^{\mathrm{b}}\left(\mathbf{k}_{M}^{\gamma}\right), \quad F \mapsto K^{J} \circ F
$$

We note that $\left.K^{\{s\}} \simeq \widetilde{K}\right|_{M \times \mathbb{R} \times M \times \mathbb{R} \times\{s\}}$. We set for short $\Psi_{s}=\Psi_{\{s\}}$. We have $\Psi_{0} \simeq$ id.

Theorem 6.1. Let $\Phi: T^{*} M \times I \rightarrow T^{*} M$ be a Hamiltonian isotopy satisfying (29). For $s \in I$ and $J \subset I$ a relatively compact subinterval let $\Psi_{J}, \Psi_{s}: \mathrm{D}^{\mathrm{b}}\left(\mathbf{k}_{M}^{\gamma}\right) \rightarrow \mathrm{D}^{\mathrm{b}}\left(\mathbf{k}_{M}^{\gamma}\right)$ be the functors defined in (85). Then for $A$ a closed subset of $T^{*} M$ and $F \in \mathrm{D}_{A}^{\mathrm{b}}\left(\mathbf{k}_{M}^{\gamma}\right)$ we have

(i) $\Psi_{s}(F) \in \mathrm{D}_{\varphi_{s}(A)}^{\mathrm{b}}\left(\mathbf{k}_{M}^{\gamma}\right)$ for any $s \in I$,

(ii) $\Psi_{[a, b[}(F)$ and $\Psi_{] a, b]}(F)$ are torsion objects for any $a<b \in I$,

(iii) for $s \in I, s \geq 0$, there exist distinguished triangles

$$
\Psi_{] 0, s]}(F) \rightarrow \Psi_{[0, s]}(F) \rightarrow F \stackrel{+1}{\rightarrow}, \quad \Psi_{[0, s[}(F) \rightarrow \Psi_{[0, s]}(F) \rightarrow \Psi_{s}(F) \stackrel{+1}{\longrightarrow}
$$

and similar ones for $s \leq 0$. In particular we have a natural isomorphism $F \simeq \Psi_{s}(F)$ in $\mathscr{T}\left(\mathbf{k}_{M}\right)$ for any $s \in I$.

Proof. (i) We set $\widetilde{\Lambda}_{s}=\widetilde{\Lambda} \circ T_{s}^{*} I$. This is the graph of $\widetilde{\varphi}_{s}$. Hence

$$
\mathrm{SS}\left(\Psi_{s}(F)\right) \cap\{\tau>0\} \subset \widetilde{\Lambda}_{s} \circ \rho^{-1}(A)=\widetilde{\varphi}_{s}\left(\rho^{-1}(A)\right)=\rho^{-1}\left(\varphi_{s}(A)\right),
$$

which proves the first statement. 
(ii)-(iii) (a) We set $\widetilde{F}=\widetilde{K} \circ F$ which belongs to $\mathrm{D}^{\mathrm{lb}}\left(\mathbf{k}_{M \times I}^{\gamma}\right)$ by Proposition 3.29, We have $\operatorname{SS}(\widetilde{F}) \cap\{\tau>0\} \subset \widetilde{\Lambda} \circ \rho^{-1}(A)$. As in Lemma 4.1 we define $A^{\prime} \subset T^{*} M \times \mathbb{R}$ by $A^{\prime}=\{(x ; \xi, \tau) ; \tau>0,(x ; \xi / \tau) \in A\}$. Then $\overline{A^{\prime}}$ is a strict $\gamma$-cone. It follows that there exists a closed strict $\gamma$-cone $B \subset T^{*}(M \times I) \times \mathbb{R}$ such that $\widetilde{\Lambda} \circ \rho^{-1}(A) \subset \widehat{\pi}^{-1}(B) \cap\{\tau>0\}$. Then Lemma 4.1 gives $\operatorname{SS}(\widetilde{F}) \subset \widehat{\pi}^{-1}(B) \cup T_{M \times I \times \mathbb{R}}^{*}(M \times I \times \mathbb{R})$. In particular $\left.\widetilde{F}\right|_{M \times J \times \mathbb{R}}$ satisfies the hypothesis of Proposition 5.9 for any relatively compact subinterval $J \subset I$.

(b) We let $q: M \times I \times \mathbb{R} \rightarrow M \times \mathbb{R}$ be the projection. For a relatively compact subinterval $J \subset I$ we have $\Psi_{J}(F) \simeq \mathrm{R} q_{*}\left(\widetilde{F} \otimes \mathbf{k}_{M \times J \times \mathbb{R}}\right)$. Then (ii) follows from Proposition 5.9, The triangles in (iii) are induced by the excision triangles associated with the inclusions $\{0\} \subset[0, s]$ and $\{s\} \subset[0, s]$. Then (ii) gives $F \stackrel{\sim}{\sim} \Psi_{[0, s]}(F) \stackrel{\sim}{\longrightarrow} \Psi_{s}(F)$ in $\mathscr{T}\left(\mathbf{k}_{M}\right)$.

\section{Application to non displaceability}

Recall that two compact subsets $A$ and $B$ of $T^{*} M$ are called mutually non displaceable if, for any Hamiltonian isotopy $\Phi: T^{*} M \times I \rightarrow T^{*} M$ satisfying (29) and any $s \in I, A \cap \varphi_{s}(B) \neq \emptyset$. A compact subset $A$ is called non displaceable if $A$ and $A$ are mutually non displaceable. Let $A$ and $B$ be two compact subsets of $T^{*} M$, let $F \in \mathrm{D}_{A}^{\mathrm{b}}\left(\mathbf{k}_{M}^{\gamma, l}\right)$ and $G \in \mathrm{D}_{B}^{\mathrm{b}}\left(\mathbf{k}_{M}^{\gamma, l}\right)$. Let $q_{2}: M \times \mathbb{R} \rightarrow \mathbb{R}$ be the projection. Recall that $\mathscr{H}_{0 m^{*}}(F, G) \in \mathrm{D}^{\mathrm{b}}\left(\mathbf{k}_{M}^{\gamma, r}\right)$ by (62). We deduce by adjunction that $\mathrm{R} q_{2 *} \mathscr{H} o m^{*}(F, G) \in \mathrm{D}^{\mathrm{b}}\left(\mathbf{k}^{\gamma, r}\right)$. We shall consider the following hypothesis:

$$
\mathrm{R} q_{2 *} \mathscr{H}{ }^{*}{ }^{*}(F, G) \text { is not torsion. }
$$

Theorem 6.2. (The non displaceability Theorem of [12, Th. 3.1].) Let $A$ and $B$ be two compact subsets of $T^{*} M$. Assume that there exist $F \in \mathrm{D}_{A}^{\mathrm{b}}\left(\mathbf{k}_{M}^{\gamma, l}\right)$ and $G \in \mathrm{D}_{B}^{\mathrm{b}}\left(\mathbf{k}_{M}^{\gamma, l}\right)$ satisfying the hypothesis (86). Then $A$ and $B$ are mutually non displaceable in $T^{*} M$.

Proof. Assume $\Phi$ is a Hamiltonian isotopy such that $\varphi_{s_{0}}(B) \cap A=\emptyset$. We consider $\widetilde{\Phi}: \dot{T}^{*}(M \times \mathbb{R}) \times I \rightarrow \dot{T}^{*}(M \times \mathbb{R})$ and $\widetilde{K} \in \mathrm{D}^{\mathrm{lb}}\left(\mathbf{k}_{M \times \mathbb{R} \times M \times \mathbb{R} \times I}\right)$ as in the introduction of this section.

We define $F^{\prime}, G^{\prime} \in \mathrm{D}^{\mathrm{b}}\left(\mathbf{k}_{M \times I}^{\gamma, l}\right)$ by $F^{\prime}=F \otimes \mathbf{k}_{I}$ and $G^{\prime}=\widetilde{K} \circ G$. We let $q_{23}: M \times \mathbb{R} \times I \rightarrow \mathbb{R} \times I$ be the projection. We have $\left.F \simeq F^{\prime}\right|_{M \times \mathbb{R} \times\{s\}}$ and we set $G_{s}=\left.G^{\prime}\right|_{M \times \mathbb{R} \times\{s\}}$. By Lemma 4.1 and Corollary 3.15, we have 
$\left.\mathscr{H}_{o m}^{*}\left(F^{\prime}, G^{\prime}\right)\right|_{M \times \mathbb{R} \times\{s\}} \simeq \mathscr{H} o m^{*}\left(F, G_{s}\right)$. By Lemma $4.1 q_{23}$ is proper on the support of $\mathscr{H}_{o m}{ }^{*}\left(F^{\prime}, G^{\prime}\right)$ and we get

$$
\left.\left(\mathrm{R} q_{23 *} \mathscr{H}_{o m^{*}}\left(F^{\prime}, G^{\prime}\right)\right)\right|_{M \times \mathbb{R} \times\{s\}} \simeq \mathrm{R} q_{2 *} \mathscr{H} o m^{*}\left(F, G_{s}\right) .
$$

Since $\operatorname{SS}\left(G_{s}\right) \subset \rho^{-1}\left(\varphi_{s}(B)\right)$, Theorem 4.3 implies $\operatorname{R}_{q_{*}} \mathscr{H}_{o m^{*}}\left(F, G_{s_{0}}\right)=0$.

By Proposition 3.13 and Lemma 3.7, the microsupport of $\mathscr{H}_{0 m^{*}}\left(F^{\prime}, G^{\prime}\right)$ is contained in $\widehat{\pi}^{-1}(C)$ for some strict $\gamma$-cone $C$. Hence a similar inclusion holds for the microsupport of $\mathrm{R}_{23 *} \mathscr{H}_{0 m^{*}}\left(F^{\prime}, G^{\prime}\right)$. Then Theorem 5.12 implies that $\mathscr{H}_{o m^{*}}\left(F, G_{s}\right)$ is torsion for all $s \in I$. In particular $\mathscr{H} o m^{*}(F, G)$ is torsion, which contradicts the hypothesis (86).

Corollary 6.3. Let $A$ and $B$ be two compact subsets of $T^{*} M$. Assume that there exist $F \in \mathrm{D}_{A}^{\mathrm{b}}\left(\mathbf{k}_{M}^{\gamma}\right)$ and $G \in \mathrm{D}_{B}^{\mathrm{b}}\left(\mathbf{k}_{M}^{\gamma}\right)$ such that $\operatorname{Hom}_{\mathscr{T}\left(\mathbf{k}_{M}\right)}(F, G) \neq 0$. Then $A$ and $B$ are mutually non displaceable in $T^{*} M$.

Proof. By Proposition 5.7, there exists $c \in \mathbb{R}$ such that the morphism induced by $\tau_{c, d}(G), \operatorname{Hom}_{\mathrm{D}^{\mathrm{b}}\left(\mathbf{k}_{M}^{\gamma}\right)}\left(F, T_{c *} G\right) \rightarrow \operatorname{Hom}_{\mathrm{D}^{\mathrm{b}}\left(\mathbf{k}_{M}^{\gamma}\right)}\left(F, T_{d *} G\right)$ is non zero for all $d \geq c$. But Lemma 3.18 gives

$$
\operatorname{Hom}_{\mathrm{D}^{\mathrm{b}}\left(\mathbf{k}_{M}^{\gamma}\right)}\left(F, T_{c *} G\right) \simeq H_{[0,+\infty[}^{0}\left(\mathbb{R} ; \mathrm{R}_{2_{*} *} \mathscr{H}_{o m^{*}}\left(F, T_{c *} G\right)\right) .
$$

On the other hand we see that $\operatorname{R} q_{2 *} \mathscr{H}_{o m}{ }^{*}\left(F, T_{c *} G\right) \simeq T_{c *} \operatorname{R}_{2 *} \mathscr{H}_{o m}{ }^{*}(F, G)$ and that $\tau_{c, d}(G)$ induces $\tau_{c, d}\left(\mathrm{R}_{q_{*} *} \mathscr{H}_{0 m^{*}}(F, G)\right)$ through this isomorphism. Hence $\mathrm{R} q_{2 *} \mathscr{H} o m^{*}(F, G)$ is non torsion and we can apply Theorem 6.2.

Let $A$ be a closed conic subset of $T^{*} M$. We know by Corollary 5.8 that the functor

$$
j_{M}: \mathrm{D}_{A}^{\mathrm{b}}\left(\mathbf{k}_{M}\right) \rightarrow \mathscr{T}\left(\mathbf{k}_{M}\right), \quad F \mapsto F \otimes \mathbf{k}_{[0,+\infty}[
$$

is fully faithful. Applying Corollary 6.3 with $F=G=j_{M}\left(\mathbf{k}_{M}\right) \in \mathscr{T}\left(\mathbf{k}_{M}\right)$ and $A=B=T_{M}^{*} M$, we get

Corollary 6.4. Assume $M$ is compact. Then $M$ is non displaceable in $T^{*} M$.

In [12, Tamarkin applies the non displaceability Theorem 6.2 to prove that the following sets are non displaceable.

Set $X=\mathbb{P}(\mathbb{C})^{n}$ endowed with his standard real symplectic structure. Consider the sets $A:=\mathbb{P}(\mathbb{R})^{n}$ and $B:=\mathbb{T}=\left\{z=\left(z_{0}, \ldots, z_{n}\right) ;\left|z_{0}\right|=\ldots\left|z_{n}\right|\right\}$. Then $A$ and $B$ are non displaceable and $A$ and $B$ are mutually non displaceable. 


\section{References}

[1] K. Fukaya, P. Seidel and I. Smith, Exact Lagrangian submanifolds in simply-connected cotangent bundles, Invent. Math. 172 p. 1-27 (2008).

[2] S. Guillermou, M. Kashiwara and P. Schapira, Sheaf quantization of Hamiltonian isotopies and applications to non displaceability problems, Duke Math. Journal, (2012). arXiv:1005.1517

[3] M. Kashiwara and P. Schapira, Micro-support des faisceaux: applications aux modules différentiels, C. R. Acad. Sci. Paris série I Math 295 8, p. 487-490 (1982).

[4] _ Microlocal study of sheaves, Astérisque 128 Soc. Math. France (1985).

[5] - Sheaves on Manifolds, Grundlehren der Math. Wiss. 292 Springer-Verlag (1990).

[6] _ Categories and Sheaves, Grundlehren der Math. Wiss. 332 Springer-Verlag (2005).

[7] R. Kasturirangan and Y.-G. Oh, Floer homology of open subsets and a relative version of Arnold's conjecture, Math. Z. 236 p. 151-189 (2001).

[8] D. Nadler, Microlocal branes are constructible sheaves, Selecta Math. (N.S.) 15 p. 563-619 (2009).

[9] D. Nadler and E. Zaslow, Constructible sheaves and the Fukaya category, J. Amer. Math. Soc. 22 p. 233-286 (2009).

[10] P. Polesello and P. Schapira, Stacks of quantization-deformation modules over complex symplectic manifolds, Int. Math. Res. Notices 49 p. 2637-2664 (2004).

[11] M. Sato, T. Kawai and M. Kashiwara, Microfunctions and pseudodifferential equations, in Komatsu (ed.), Hyperfunctions and pseudodifferential equations. Proceedings Katata 1971, Lecture Notes in Math. Springer-Verlag 287 p. 265-529 (1973).

[12] D. Tamarkin, Microlocal conditions for non-displaceability, arXiv:0809.1584 
Stéphane Guillermou

Institut Fourier

Université de Grenoble I

BP 74, 38402 Saint-Martin d'Hères, France

email: Stephane.Guillermou@ujf-grenoble.fr http://www-fourier.ujf-grenoble.fr/ guillerm/
Pierre Schapira

Institut de Mathématiques

Université Pierre et Marie Curie

4, place Jussieu, case 247, 75252 Paris cedex 5,

France

e-mail: schapira@math.jussieu.fr

http://www.math.jussieu.fr/ schapira/ 